\title{
The Clinical Utility of Genetic Risk Scores Following Myocardial Infarction
}

\author{
Hadley Northcott
}

\author{
A thesis \\ submitted to Victoria University of Wellington \\ in fulfilment of the requirements for the degree of \\ Master of Biomedical Science
}

Victoria University of Wellington

2016 
Hadley Northcott 


\section{Abstract}

Current risk assessment for the development of coronary artery disease (CAD) in an individual relies on a combination of clinical characteristics. These well-established CAD risk factors include consideration of age, gender, hypertension, dyslipidemia, diabetes, smoking and obesity. However there are a proportion of patients that experience an acute coronary syndrome (ACS) event despite being deemed as low risk based on the current New Zealand risk model. These patients present with an absence of the traditional risk factors, or they fall below the age threshold where CAD screening is initiated.

The lack of association with disease development and presence of the traditional risk factors in these patients has led to the hypothesis that genetics play a significant role in the etiology of their disease. The conduction of family-based hereditary studies has supported the hypothesis that CAD risk is associated with genetic markers. A method of analyzing this genetic risk has been developed in the form of calculating a genetic risk score (GRS). The GRS is comprised of a panel of single nucleotide polymorphisms (SNPs) discovered through genome wide association studies in CAD patients. Currently, there is controversy in the clinical utility of different GRS calculation methods, and as yet, there has been no research conducted on the potential benefits of a GRS in a New Zealand setting.

Our study measured genetic risk through a weighted GRS calculated from a 27 SNP panel in 420 patients in a New Zealand based population. In looking at whether we could determine a difference in GRS values between premature (young) MI patients and older control patients, we found that the mean GRS was not significantly elevated in the premature MI cohort $(\mathrm{p}=0.156)$. However, in assessing GRS differences between ethnicities and in relation to specific risk factors we saw that mean GRS was higher in patients with a family history of coronary disease ( $\mathrm{p}=0.003)$, in Māori patients $(\mathrm{p}=0.013)$ and in patients with fewer than 2 traditional risk features ( $p=0.001)$. GRS was not associated with individual traditional risk factors, including dyslipidaemia, hypertension, diabetes, obesity or gender. Our results showed that genetic risk for CAD is identifiable with this GRS, and indicates that further research into ethnic differences and identifying genetic risk in young CAD patients with low traditional risk would provide interesting insights. 


\section{Acknowledgements}

To my supervisors, Associate Professor Peter Larsen and Dr. Scott Harding, thank you for all of your guidance and for the opportunities you have given me. Without your mentoring this thesis would not have been possible.

To the team in the VUW Clinical Research Laboratory, thank you for your generous help and advice, for helping me find my feet in this new setting, and for keeping me laughing throughout.

To my friends, for your encouragement, and for keeping me sane. Thank you for keeping my life outside of thesis writing interesting.

And to my family, the best support team anyone could ask for. Mum, Dad, Cerise, Adam, my grandparents, the list goes on. Thank you for keeping me motivated, keeping me alive, and for riding the ups and downs with me. I wouldn't have made it here without you, thank you. 


\section{Table of Contents}

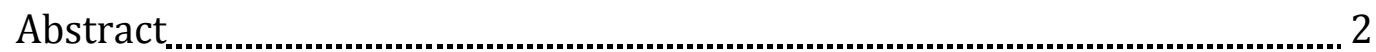

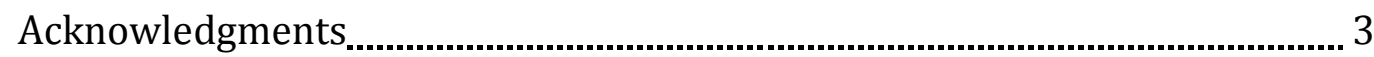

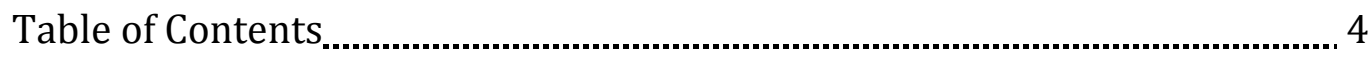

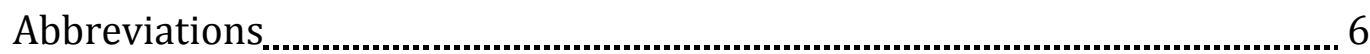

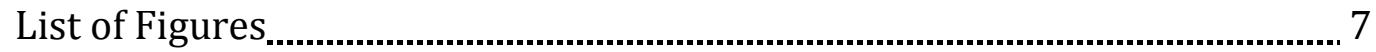

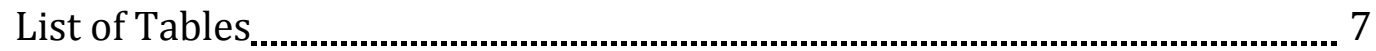

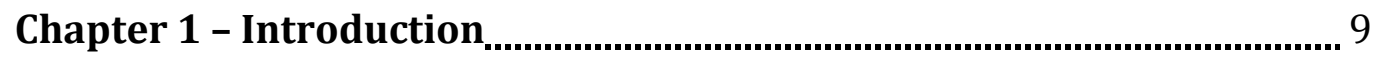

1.1 Coronary Artery Disease ................................................................. 9

1.1.1 Premature MI............................................................................. 10

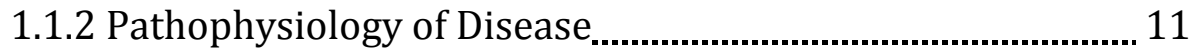

1.2 Acute Coronary Syndromes (ACS) ...................................................... 14

1.2.1 Clinical Management of ACS................................................. 14

1.3 Traditional Risk Factors ................................................................. 16

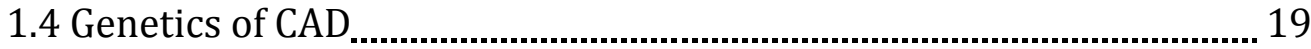

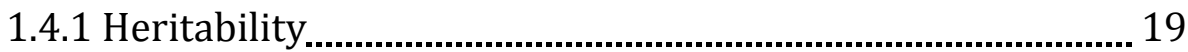

1.4.2 Known Genetic Risk Factors .................................................... 19

1.4.3 Genome Wide Association Studies........................................ 20

1.4.4 SNP Panels and Genetic Risk Scores..................................... 21

1.4.5 The SNP Panel ........................................................................... 24

1.4.6 SNP Panel Gene Functions ..................................................... 26

1.5 Thesis Aims

Chapter 2 - Methods..................................................................................... 33

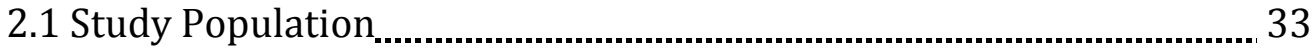

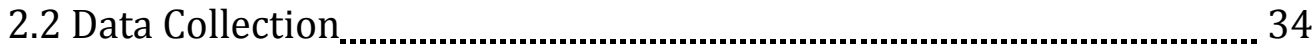

2.3 Sample Collection and Storage ........................................................... 34

2.4 DNA Extraction ................................................................................ 34

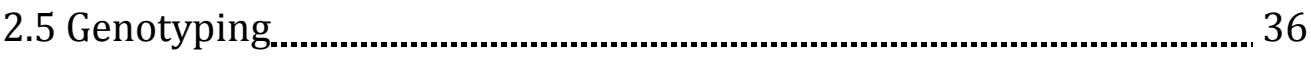

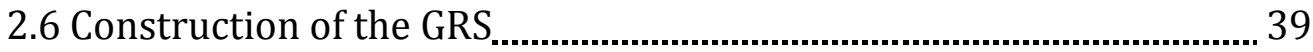

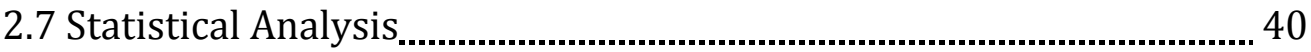




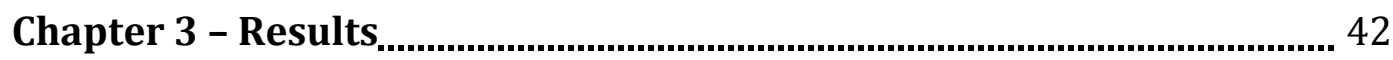

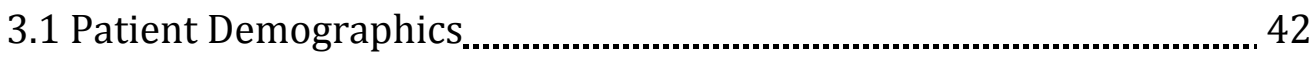

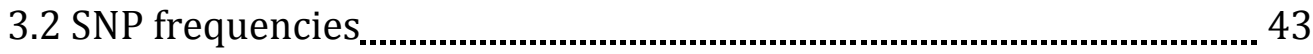

3.3.1 Between Premature MI and Over 60 MI.................................. 43

3.3.2 Between Māori and non-Māori................................................ 45

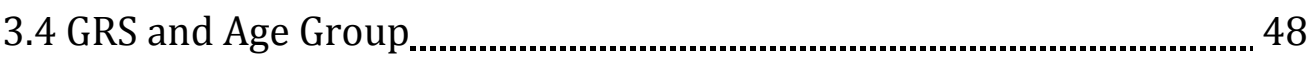

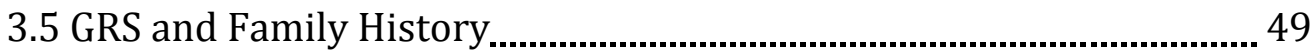

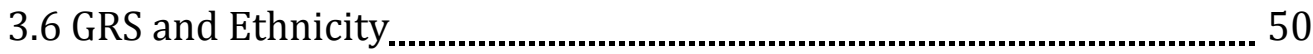

3.7 GRS and Risk Factors ............................................................................ 51

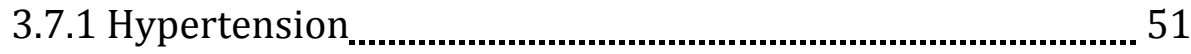

3.7.2 Dyslipidemia ........................................................................ 52

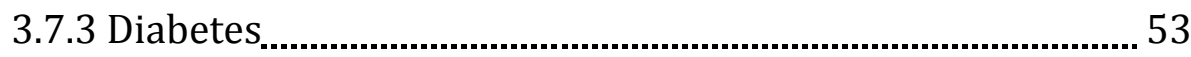

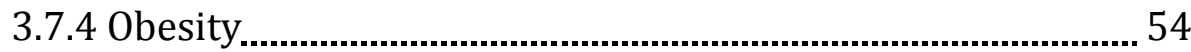

3.7.5 Combined Risk Factors .............................................................. 55

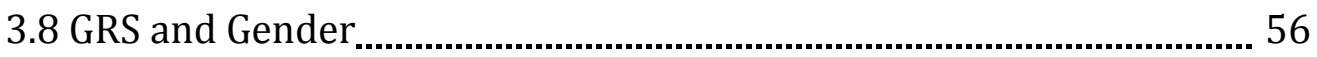

Chapter 4 - Discussion .......................................................................... 58

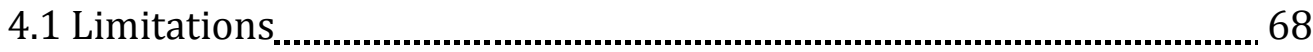

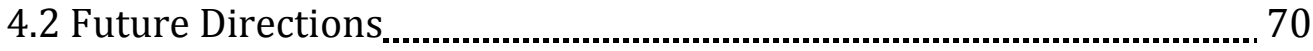

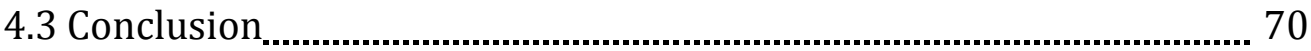

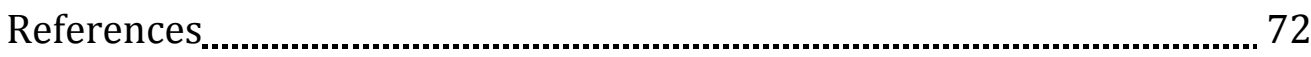




\section{Abbreviations}

ACS - acute coronary syndrome

BMI - body mass index

CAD - coronary artery disease

CVD - cardiovascular disease

DAPT - dual anti-platelet therapy

ECG - electrocardiogram

GRS - genetic risk score

GWAS - genome wide association study

HDL - high density lipoprotein

HR - hazard ratio

LDL - low density lipoprotein

MI - myocardial infarction

NSTEMI - non-ST-segment elevation myocardial infarction

NZ - New Zealand

OR - odds ratio

PCI - percutaneous coronary intervention

SNP - single nucleotide polymorphism

STEMI - ST-segment elevation myocardial infarction

UA - unstable angina 


\section{Figures}

Figure 1: Progression of the atherosclerotic plaque .............................................. 13

Figure 2: New Zealand cardiovascular disease risk assessment chart ..................... 16

Figure 3: SNP chromosome ideogram ................................................................... 25

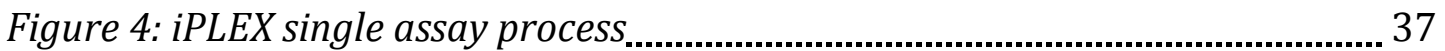

Figure 5: Risk allele SNP frequencies between study groups.................................. 43

Figure 6: Risk allele SNP frequencies in Mãori and non-Māori................................. 45

Figure 7: Distribution of GRS values in the total study population ...........................48

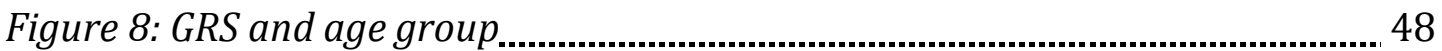

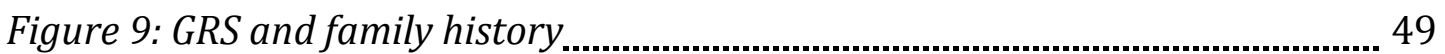

Figure 10: GRS and age group in patients with family history .............................. 49

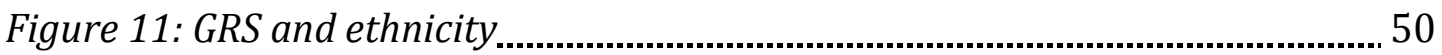

Figure 12: GRS and hypertension ....................................................................... 51

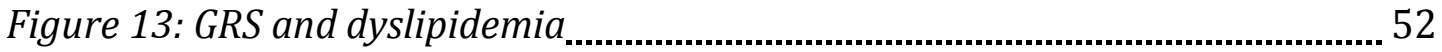

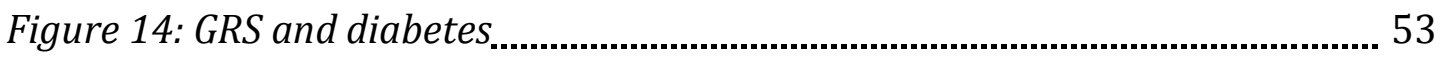

Figure 15: GRS and obesity ................................................................................ 54

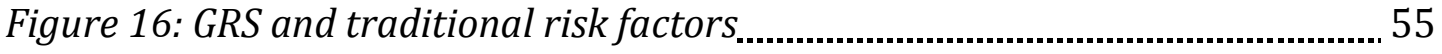

Figure 17: Mean number of risk factors per GRS quintile ....................................... 55

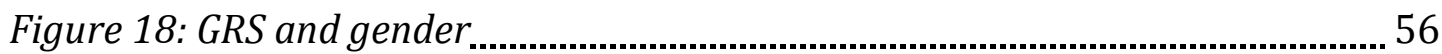

\section{Tables}

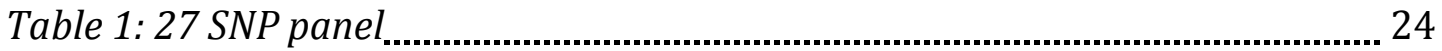

Table 2: Clinical characteristics of the study population ........................................... 42

Table 3: Risk allele SNP frequencies between study groups.................................... 44

Table 4: Risk allele SNP frequencies in Māori and non-Māori................................ 47 
Hadley Northcott 


\section{Chapter 1 - Introduction}

\subsection{Coronary Artery Disease}

Coronary Artery Disease (CAD) is the single leading cause of death and morbidity worldwide ${ }^{1,2}$. In 2012, deaths directly attributed to CAD numbered 7.4 million, accounting for $42 \%$ of all cardiovascular disease deaths ${ }^{2}$. In the US alone, someone experiences a myocardial infarction (MI) every 43 seconds and of the people that experience a coronary event approximately 34\% will die because of it $^{3}$. It is estimated that the cost of heart disease in the US in 2010 was over USD\$204 billion ${ }^{3}$.

In New Zealand, in alignment with the rest of the developed world, CVD is a leading cause of death and morbidity, with CAD making up the greatest proportion of these. CAD accounted for 5339 deaths in 2012, which was $17.6 \%$ of all deaths. A New Zealander dies from heart disease every 98 minutes $^{4} .4 .6 \%$ of New Zealand adults have been diagnosed with CAD, numbering approximately 169,000 people ${ }^{5}$, with a significant number of individuals estimated to be living with undiagnosed CAD. The economic cost of CAD accounts for a large percentage of New Zealand health spending and puts significant strain on the health system.

The New Zealand population has a unique ethnic diversity, with the indigenous Māori population being over represented in national mortality and morbidity statistics ${ }^{6}$. Māori MI rates are seen to be consistently high, at a level that is disproportionate to the rest of the NZ population. Māori ischaemic heart disease mortality rates are 106.2 per 100,000 population, compared to that of non-Māori at 58.04. While researchers have often hypothesized that there may be a genetic link to the increased rate of MI in Māori, a common argument has been that environmental and socioeconomic factors play more of a role ${ }^{7}$. These include factors at the individual level, such as diet and exercise, as well as population level inequalities with access to the healthcare system, with Māori being less likely to be referred for some cardiac interventions than non-Māori 8,9.

Māori health initiatives are currently underway in order to ameliorate these ethnic healthcare differences $^{6}$. A large component of this is addressing individual cardiac risk factors, of which a number are observed at a higher rate in Māori. Māori have a higher percentage of smokers and 
obesity than non-Māori and also have much higher rates of mortality from hypertension and diabetes ${ }^{4,5}$.

\subsubsection{Premature MI}

CAD is often considered a disease of the elderly, however a small proportion of MI events occur in patients that are younger than 50 years old. Previous studies undertaken in international cohorts have assessed the incidence of premature MI to be between 2-10\%10-20. In New Zealand, premature MI was observed to account for $12.8 \%$ of cases in the Wellington Acute Coronary Syndrome Registry (WACSR), in a study involving 1199 MI patients ${ }^{21}$. The risk profile of patients that experience premature MI is often observed to differ from that of older MI cases. In the WACSR study, the premature MI group were more likely to be male, of Māori or Pacific Island ethnicity, have a higher BMI, have a family history of CAD, and to be current smokers. The older MI patients were more likely to have hypertension, dyslipidemia and diabetes ${ }^{21}$. Age is often used as a strong indicator of CAD risk ${ }^{22}$, however these studies outline that relying upon age as a key risk factor will exclude a significant proportion of at risk patients. Within the group of patients who experience premature MI, a small subset of patients show little or no risk according to traditional risk factors. In the WACSR study 36\% of patients had one or fewer traditional risk factors. These patients would have been classified as low risk for cardiovascular disease using current guidelines ${ }^{22}$. Thus, we can see that there are complex factors at play that are not completely captured by current CAD risk assessment methods. The use of genetic screening, such as genetic risk scoring, looks to aid in addressing this issue.

Although a higher proportion of premature MI cases are seen to be caused by nonatherosclerotic processes, such as coronary abnormalities, hypercoagulable states and recreational drug use than in older MI patients, CAD processes are still seen in the majority of cases $^{15}$. In the case of the WACSR study, all patients that were classified as unstable angina or had an alternative diagnosis that was not consistent with MI were removed ${ }^{21}$. 


\subsubsection{Pathophysiology of Disease}

CAD is a condition in which plaques accumulate within the coronary vessels, leading to restricted blood flow to the heart. CAD is well regarded as a progressive inflammatory disease. It presents as plaques within the coronary arteries, which in advanced stages can become large enough to inhibit blood flow 23,24 . The most severe complications normally arise due to rupture of the plaque, forming a thrombus ${ }^{25}$.

Coronary artery lesions, or atherosclerotic lesions, are believed to initiate through the process of lipids, namely low density lipoprotein (LDL), accumulating within the subendothelial matrix of the endothelial cells of artery walls. LDL is able to diffuse through the endothelial lining of the arteries, where it undergoes modifications, such as oxidation, which enables the LDL to be up-taken by macrophage cells $23,26,27$.

LDL oxidation takes place primarily through the action of reactive oxygen species (ROS) such as the superoxide radical $\left(\mathrm{O}_{2}^{-}\right)$, hydroxyl radical $(-\mathrm{OH})$ and hydrogen peroxide $\left(\mathrm{H}_{2} \mathrm{O}_{2}\right)$. ROS production is increased by some of the traditional risk factors for CAD, such as hypercholesterolemia and smoking, which have adverse effects on endothelial function ${ }^{28}$. As well as oxidative modifications on LDL, an increase in ROS can lead to a decrease in NO, which subsequently can result in impaired vasorelaxation and reduce NO's inhibitory effects on platelet and leukocyte adhesion, and create a proinflammatory environment. Sources of ROS include nicotinamide adenine dinucleotide phosphate (NADPH), oxidases, xanthine oxidases, enzymes of the mitochondrial respiratory chain, and endothelial nitric oxide synthase $(\mathrm{eNOS})^{28,29}$.

The sequestering of the now proinflammatory lipoprotein in the endothelium results in immune cell recruitment, namely monocyte derived cells such as macrophages. The lipoprotein is phagocytized by macrophages, forming lipid-laden foam cells. These in turn recruit other immune cells and proinflammatory mediators, including chemokines and cytokines, as well as stimulating the proliferation of vascular smooth muscle cells (VSMC). These processes form the nascent atherosclerotic lesion. In a stable atherosclerotic lesion, proliferation of VSMC and the subsequent increase in fibrous tissue, i.e. collagen and extracellular matrix ${ }^{28,30}$, forms a fibrous cap over the central, necrotic core of foam cells. Lesion progression can inhibit arterial blood 
flow by luminal narrowing. The release of matrix-degrading enzymes, through the increased release of cytokines resulting from the apoptosis of VSMC, erodes the fibrous cap ${ }^{28}$. The decrease in integrity of the fibrous cap leaves the plaque increasingly susceptible to rupture ${ }^{31}$.

Plaque rupture, believed to occur mostly due to mechanical factors, such as blood flow, at sites of weakness in the fibrous cap exposes the core of the lesion 27,32 . The lipid core of the ruptured plaque is often highly thrombogenic, and when this is exposed to the thrombogenic components of blood, thrombus formation occurs ${ }^{27}$, often reducing the flow of blood to the heart muscle. Thrombi can form due to platelet activation and activation of the coagulation cascade due to the presence of tissue factor and fibrin in the ruptured plaque. The clinical manifestations of these processes present as acute coronary syndromes (ACS), including unstable angina (UA) and myocardial infarction (MI). 


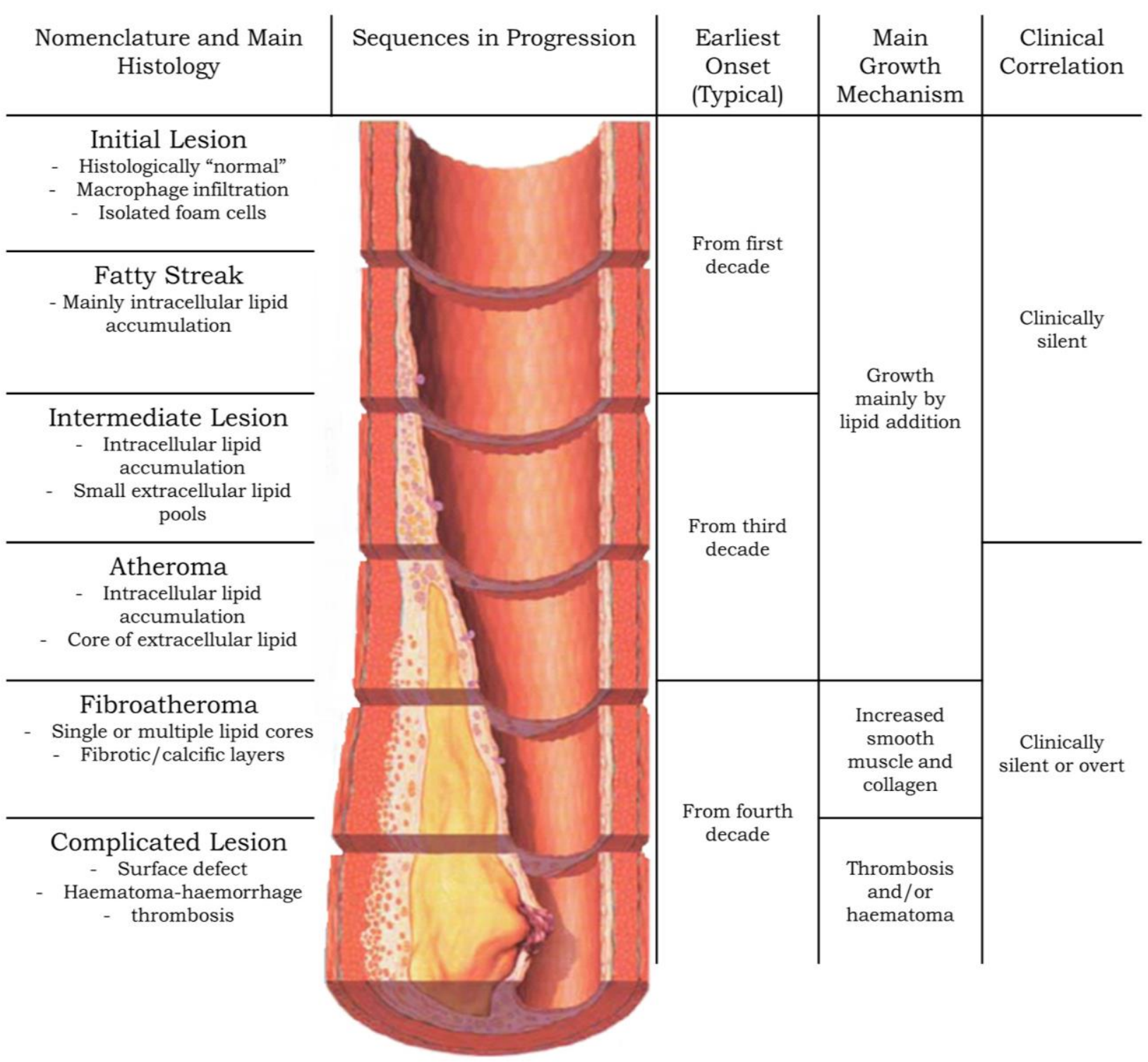

Figure 1: Progression of the atherosclerotic plaque.

A simplified depiction of the sequences in atherosclerosis progression, showing main histology, typical age of onset (this differs in premature MI), growth mechanism and clinical correlation. Adapted and reproduced with permission from Creative Commons Attribution Share. Transferred from en.wikipedia to Commons., CC BY-SA 3.0, https://commons. wikimedia.org/w/index.php?curid=2816579 


\subsection{Acute Coronary Syndromes}

The unstable presentation of CAD is defined as an acute coronary syndrome (ACS). An ACS event is most often due to rupture of an unstable plaque, resulting in the formation of a thrombus which occludes blood flow to a portion of the heart. This can be a life threatening condition. This occlusion of blood flow in the arterial lumen can result in ischaemia and subsequent infarction of the cardiomyocytes of the heart ${ }^{25}$. ACS symptoms can occur without detectable cardiac necrosis. The ACS manifestation of this occurs when these symptoms are present even when the patient is at rest, with no cardiac cell death being detected, and is termed unstable angina (UA). UA events are rarely fatal, however are a strong indicator of the presence of a high grade stenosis and normally do require treatment, either through clinical intervention or medical management. The formation of a thrombus very often leads to myocardial infarction (MI), which involves at least some degree of cell death within the heart (infarction). An occluding thrombus can be fatal, even when resolved. Where the degree of infarction within the heart wall is significant, structural problems can arise, which can lead to cardiac remodeling and arrhythmias ${ }^{25}$. Thrombus formation is most likely to occur on vulnerable plaques, i.e. plaques with thin fibrous caps that are therefore more susceptible to rupture ${ }^{31}$.

\subsubsection{Clinical Management of ACS}

UA is defined as symptoms of ischaemia at rest with no associated cardiomyocyte necrosis, as determined by levels of cardiac troponin ${ }^{33}$. MI is defined as a rise in cardiac troponin concomitant with either symptoms of ischaemia (e.g. chest pain) and/or ECG changes. An MI can be further categorized as a non-ST-elevation myocardial infarction (NSTEMI) or a STsegment elevation myocardial infarction (STEMI). Patients with acute chest pain and a rise in troponin levels, but with no persistent ST-segment elevation on a 12-lead electrocardiogram (ECG) characterize a NSTEMI. A STEMI is characterized by acute chest pain, troponin increases and persistent ST-segment elevation. The difference in NSTEMI and STEMI, shown by STsegment changes $\geq 0.1 \mathrm{mV}$ on a 12-lead $\mathrm{ECG}^{34}$, is brought about due to the degree of ischaemia in the wall of the heart. An ST-segment elevation is indicative of full thickness ischaemia in the heart wall; that is a transmural injury, which is perceived to be more severe than a subendocardial infarction. 
The primary goal when treating patients that present with ACS events is to limit the extent of necrosis that occurs within the heart and to mitigate the damage that has already taken place. Upon presentation, serial ECGs and biomarker levels (troponins) should be taken, along with a complete set of vital signs. Aspirin should be given to patients with suspected ACS unless contraindicated, and nitroglycerin or analgesia can be given if required to ameliorate acute symptoms. Percutaneous coronary intervention (PCI) and thrombolysis are the primary treatments for reperfusion. Standard medications that are often administered and prescribed following discharge include anti-platelet drugs such as clopidogrel, which is an irreversible $\mathrm{P}_{2} \mathrm{Y}_{12}$ inhibitor and requires hepatic activation, or ticagrelor, which is a reversible $\mathrm{P}_{2} \mathrm{Y}_{12}$ inhibitor that does not require hepatic activation. Other medications can include beta-blockers, anticoagulants (such as heparin), and statins ${ }^{35}$.

The most robust method of reperfusion during an ACS is through PCI, which takes place during a coronary angiogram. An angiogram involves the insertion of a catheter through either the radial or femoral artery as an access point to the coronary arteries. Guidelines for treatment with PCI differ between NSTEMI and STEMI cases ${ }^{36,37}$. Assuming the degree of CAD present is treatable through stenting, angioplasty or medical management, and coronary artery bypass grafting (CABG) is not required, the standard hospital stay for an ACS patient need not exceed a few days. 


\subsection{Traditional Risk Factors}

There are a number of well-established risk factors for CAD, which are assessed during cardiovascular risk scoring. Initially, these risk factors were observed and incorporated into a risk-screening framework during the Framingham Heart Study (FHS). The FHS is an ongoing cohort study that began in 1948, looking at the epidemiology and risk factors of cardiovascular disease $^{38}$. Recognized risk factors for cardiovascular diseases include hypertension, dyslipidaemia, diabetes, smoking and obesity with gender differences and family history known to influence risk as well 22 .

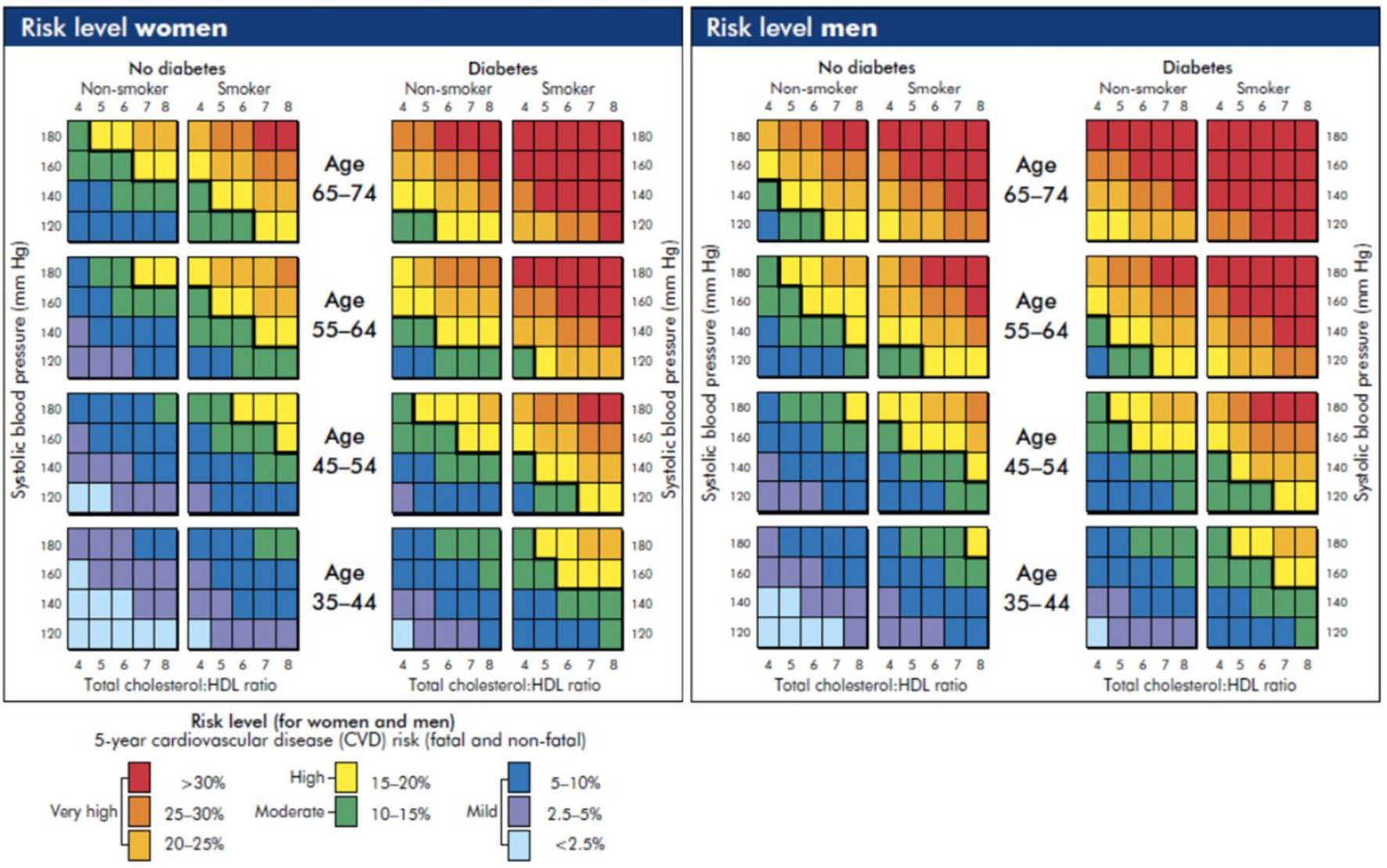

Figure 2: New Zealand Cardiovascular Risk Assessment Chart.

Reproduced with permission from New Zealand Ministry of Health under a Creative Commons Attribution 4.0 International License 
Hypertension is a well-established risk factor of CAD that contributes to the progression of atherosclerotic lesions through a number of mechanisms, including damage to the endothelial lining of arteries and increases in oxidative stress in the arterial endothelium, which activates inflammatory responses. However, the exact processes involved are yet to be entirely elucidated $^{39}$. Thiazide diuretics, beta-blockers, angiotensin-converting enzyme inhibitors, angiotensin II receptor blockers, calcium channel blockers and renin inhibitors are all used to treat hypertension.

Another factor in the development of atherosclerosis is dyslipidemia. High LDL and low highdensity lipoprotein (HDL) levels are known to lead to increased risk of CAD and subsequent $\mathrm{MI}^{40,41}$. Higher circulating levels of LDL result in more LDL molecules being available to accumulate within atherosclerotic lesions. Lower circulating levels of HDL results in a decrease of HDL's effects in inhibiting the oxidation of LDL molecules ${ }^{42}$. There are medications available that have been largely successful at controlling cholesterol levels. Statin drugs, one of the most highly consumed medications in the world, act as HMG-CoA reductase inhibitors, limiting the production of cholesterol in the mevalonate pathway. In this way they decrease LDL levels, and even have modest effects in increasing HDL concentrations, which has been seen to be beneficial in the CAD setting ${ }^{43,44}$. Other medications, such as PCSK9 inhibitors also decrease cholesterol levels, albeit through alternative mechanisms ${ }^{45}$. Lipoprotein (a) [Lp(a)], a lipoprotein with an LDL-like moiety, has also been causally linked to increased CVD risk. Research into the impact of $\mathrm{Lp}(\mathrm{a})$ on atherothrombotic risk is ongoing, however it is increasingly recommended as a beneficial biomarker in assessing CAD risk. Lp(a) is also largely believed to be controlled mostly through genetics, opening up new challenges in relation to treatment 46,47 .

Another major contributor to CAD and ACS events is diabetes. CAD is the largest cause of mortality in patients with diabetes mellitus. Diabetics are more than twice as likely to develop cardiovascular disease ${ }^{48,49}$ and the presence of diabetes is seen to result in a higher proportion of fatal $\mathrm{MI}^{49}$. Also, traditional risk factors do not account for the entire association between diabetes and CAD, therefore it is believed that there are other mechanisms that are also contributing 49 . 
Two modifiable risk factors are obesity and smoking. Obesity is a well-established risk factor for CAD, both indirectly through covariates, such as hypertension, dyslipidaemia, reductions in HDL and non-insulin dependent diabetes mellitus, as well as being an independent predictor of CAD in itself ${ }^{50}$. Obesity is a global epidemic of increasing concern, thus many health initiatives are working to mitigate the increasing rates of obesity worldwide ${ }^{51}$. Obesity is generally defined as a body mass index $(\mathrm{BMI}) \geq 30$; however, this definition raises concern as it does not take body fat distribution into account ${ }^{50}$. Smoking is a contributor to a number of disease states. In the CAD setting, it is believed to play a role in lesion progression through interactions with the arterial wall as well as influencing levels of lipoproteins and circulating platelets ${ }^{52}$.

Gender is known to play a role in CAD risk, and this is factored in during risk screening. The incidence of ACS events, especially in patients under 50 years of age, is more prevalent in males than in females. Women tend to suffer from a primary ACS event approximately 10 years later than men, and also tend to experience worse outcomes ${ }^{53}$. Age is a key factor that is used in CAD risk assessment. A majority of ACS events occur after the age 50, with a drastic increase in events being seen in the NZ population after 35 years of age. The prevalence of CAD is higher in NZ males compared to females in all age groups ${ }^{7}$.

There are a small proportion of patients that experience an MI even though they do not possess any of the traditional risk factors for CAD. For these patients it seems that there are processes involved in the development of CAD that are yet to be discovered, and thus escape current means of risk screening. A popular hypothesis for this is that a significant proportion of this risk is driven by genetic factors. It has been proposed that genetic variations may increase an individual's susceptibility to each or a combination of traditional risk factors, which can also then be exacerbated by environmental factors, as well as some genetic variation that increases an individual's risk by acting in processes not currently accounted for by traditional risk factors. It is well documented that family history is an important aspect of determining CAD risk $^{54,55}$ and while some of this may be accounted for by environmental factors shared within a family, genetics is also believed to play a significant role. 


\subsection{Genetics of CAD}

\subsubsection{Heritability}

It is estimated that between $30-60 \%$ of the variation in CAD risk is attributable to genetic factors $^{54,56,57}$. Familial clustering of CAD has been well documented, especially in studies of twins $54,57-59$. Marenberg et al concluded that death from CAD in younger individuals is likely to be influenced by genetic factors, and that the genetic effect decreases with age ${ }^{54}$. It has also been observed that the presence of CVD in a parent is an independent risk factor for CVD risk in offspring and, after adjustment for other risk factors, a significant proportion of this was deemed to be due to genetics ${ }^{55,60}$. Parental history was not only found to be an indicator of the presence of CAD in offspring, but also, for patients with low levels of traditional risk factors, indicated the extent of the occlusions found during coronary angiography to be greater 61 .

\subsubsection{Known Genetic Risk Factors}

The etiology of CAD is known to be multi-faceted, not only with the number of environmental factors, but also with the vast number of genetic factors that play a role. The interactions between the environmental and genetic factors are complex. An individual may inherit the version of a gene that makes them more susceptible to CAD, but it often involves specific environmental factors to interact with the person's specific genotype for the disease to progress $^{62}$. Therefore, in the vast majority of patients we do not see CAD being present due to a single Mendelian trait. However, there are rare cases, most often in familial clusters, where a single gene has been identified as the major cause of an individual's CAD.

Thanks to candidate gene and linkage analyses studies there is a list of established genetic mutations that are well studied in regards to their mechanisms in CAD development. Some of these are single gene mutations that impact key processes. Modifications in one such gene, an autosomal dominant mutation in $L D L R$, coding for the LDL receptor, causes familial hypercholesterolemia (FH). FH is the result of the LDL receptors diminished ability to control circulating LDL levels. Patients with FH are much more likely to suffer an ACS at a younger age due to accelerated development of atherosclerotic lesions ${ }^{63}$. Familial defective mutations in the 
apolipoprotein $\mathrm{B}-100$ gene $(A P O B)$ can result in a clinical phenotype that is indistinguishable from the FH that results from $L D L R$ mutations ${ }^{62}$.

$M E F 2 A$, a gene believed to cause an autosomal dominant form of CAD (adCAD1) is found on chromosome 15 and codes for a transcription factor that is thought to play a role in myocyte differentiation ${ }^{62}$. This MEF2A signaling pathway was implicated in CAD pathogenesis during a study of one large family, which included 13 members with CAD, and who shared a 7 amino acid deletion in the $M E F 2 A$ gene ${ }^{64}$.

These genes are an example of a group of known genetic causes of CAD that often act as the primary source in the pathology of the disease. The presence of mutations within these genes often resulting in large effects by themselves, as well as the fact that these mutations are relatively rare, results in them offering little benefit for use within a SNP panel for a GRS. These GRS' are designed to calculate risk of CAD due to the combined effects of a number of mutations in the general population, and to find this risk where it might normally be missed. Where these mutations are present, their effects will normally be discovered from the more severe phenotypes that result, hence their inclusion in a GRS is often not required. These factors do not account for all of the genetic risk that is associated with CAD. There are known to be many more genes, involved in many more processes, including both genes and processes that are yet to be elucidated within the CAD context.

\subsubsection{Genome Wide Association Studies}

A key method in discovering these genetic markers is the use of genome wide association studies (GWAS). GWAS is a powerful tool for analyzing DNA sequence variations across the entire genome, in search of genetic risk factors for common diseases. The unit of variation analyzed in GWAS is the single nucleotide polymorphism (SNP), which is a change in the genome that involves just one base pair65. GWAS typically compare thousands of different SNPs across thousands of patients, using the common disease-common variant hypothesis. Numerous GWA studies have taken place within the CAD context, leading to the discovery of many SNPs that are linked to $\mathrm{CAD}^{66-68}$. Of these studies, a number of meta-analyses have been run, producing confirmation of existing SNPs as well as further discovery of novel loci69,70. 


\subsubsection{Single Nucleotide Polymorphism Panels and Genetic Risk Scores}

The individual SNPs discovered in these GWA studies, although having reached significance in their association with CAD, often only have modest effects in their actual contribution to the disease. For this reason, panels incorporating a number of stringently validated SNPs are produced. These SNP panels aim to assess the combined effects that the presence of the SNPs may have on an individual through the calculation of a genetic risk score (GRS). The use of a GRS proposes many benefits, in the form of the sheer scale of variants that can be analyzed, the precision in disease etiology and therefore treatment strategies it offers and the new pathways that are implicated in the disease process that can be discovered. A major benefit also, is that genetic information is consistent through the lifespan of an individual, therefore a GRS can complement risk screening at any stage ${ }^{56}$.

Many studies have incorporated the use of a GRS in order to assess the combined genetic effects of different combinations of SNPs on CAD and MI. The majority of studies identify that a GRS, in whichever way it was constructed, associates with CAD and/or MI risk. However, the additive information that the GRS generates does vary, and therefore there are mixed results in regards to the power of the GRS to give extra risk prediction over and above traditional risk factors ${ }^{71-87}$. While all of these studies were similar in their design and methodology, using GRS calculations based upon the presence or absence of SNPs found to be associated CAD, a number of differences exist between them. The SNPs incorporated into the GRS differed greatly between the studies. Some focused on single pathways believed to be crucial in CAD onset ${ }^{73,77}$, while others were much more broad 71,86 . The number of SNPs used varied significantly, ranging from 9 to $49,310^{71,88}$. Also, the patient cohorts used included different demographics and ethnicities. In addition to this, the method of calculating the GRS can also change, with some studies using weighted sums of the alleles present and others simply adding the number of risk alleles seen. Consequently we see substantial disagreement in the literature in regards to which method of GRS and which combination of SNPs is most beneficial in increasing CAD risk prediction. Therefore, more research is required to test these GRS study designs in different populations.

While the number of SNPs that can be incorporated into a GRS can vary immensely, a range of SNPs from 10-50 seems to show sufficient utility, with panels bigger than this often not producing significantly increased benefit. One study, conducted by Vaara et al, tested this 
hypothesis by comparing the GRS of two SNP panels in estimating recurrent ACS. One incorporated 47 SNPs (GRS47) and the other 153 SNPs (GRS153). Their results showed that GRS47 showed association with recurrent ACS, whereas GRS153 did not ${ }^{86}$. Another example of this is found in the studies of Abraham et al and Kathiresan et al. Abraham et al used a GRS calculated from a panel of 49,310 SNPs covering a broad range of cellular processes and found that this GRS did associate with incident ACS events independent of traditional risk factors ${ }^{71}$. At the other end of the scale, Kathiresan et al used a GRS calculated from 9 SNPs that are all associated with LDL or HDL levels. They were able to see an association between their GRS and incident cardiovascular disease (outcomes included myocardial infarction, ischaemic stroke and death), that similarly was independent of traditional risk factors, even though they had 49,301 less SNPs ${ }^{88}$. Hence we can see that the quality of a SNP panel does not appear to rely on the number of SNPs analyzed, but more on what specific SNPs are included.

Mega et al calculated a GRS from the presence or absence of 27 SNPs in their panel. The number of risk alleles present $(0,1$ or 2$)$ was weighted by the log of the odds ratio for each of the SNPs. A linear sum of these then produced the genetic risk score for each patient. They tested to see whether this GRS could identify risk of incident and recurrent ACS events in 48,421 patients from five different studies. They found that their GRS could identify individuals who were at increased risk for both incident and recurrent ACS events ${ }^{89}$. The results of this study, with the successful identification of ACS risk in nearly 50,000 European patients, inspired the basis of this thesis, with the aim of assessing the GRS score derived from Mega et al within the New Zealand context. NZ has a unique ethnic diversity, and the similarities of risk markers when compared to European populations are unknown.

This current thesis set to explore the variation in genetic risk between patients who experienced premature MI and those that suffered an MI at age 60 or older within a local New Zealand based population, involving CAD patients presenting to Wellington Hospital. This is designed to help answer whether the SNPs identified in GWAS studies to date, namely the 27 SNPs within the Mega et al panel, are involved in processes that exacerbate the earlier onset of $\mathrm{CAD}$, or if they are more strongly associated to the majority of CAD cases that occur in later life. Using the GRS technique, we will also be able to see the spread of GRS values throughout the population, and be able to equate risk with specific clinical characteristics. 
The number of studies involving GRS methodology have all taken place in populations different from New Zealand's unique ethnic groups. As such, they are have unknown utility in the NZ healthcare setting, as our Māori population may show substantial variation in risk alleles and SNP frequency. Therefore, in order to gather the information required to one day include more personalized genetic risk screening in to our CAD prevention guidelines, research such as this is required to assess whether studies conducted overseas in mostly European and US patients are able to be extrapolated for use in NZ. The design of this study will also enable us to gather pilot data for questions regarding SNP frequencies in our population, and the relationship between traditional risk factors (individual and combined) and genetic risk.

The study will incorporate the 27 SNP panel, designed by Mega et al from SNP data generated from the Malmo Diet and Cancer study (MDCS) ${ }^{90}$. Genetic risk will be scored in the same manner as within the Mega et al study also, calculating the sum of the weighted SNPs that are present. The SNPs included in the panel were chosen by Mega et al on the basis of their significant association with $\mathrm{CAD}$ at a genome wide level, and their subsequent validation in further studies. The results of the Mega et al study show that the use of this specific SNP panel and GRS calculation does have the ability to detect increased genetic risk in both primary and secondary prevention in Europeans. The overarching goal of this theme of study is to further the research towards precision medicine. In being able to assess an individual's genetic risk, and use this in conjunction with traditional risk factor screening, the possibility to tailor treatments and preventative strategies becomes more of a realistic objective. 
1.4.5 The SNP Panel

\begin{tabular}{|c|c|c|c|c|}
\hline Lead SNP & Position (Gene) & Risk Allele & OR for CAD & Risk Frequency \\
\hline rs646776 & 1p13.3 (SORT1) & $\mathrm{T}$ & $1 \cdot 19$ & $0 \cdot 77$ \\
\hline rs17114036 & 1p32.2 (PPAP2B) & A & $1 \cdot 17$ & 0.92 \\
\hline rs11206510 & 1p32.3 (PCSK9) & $\mathrm{T}$ & $1 \cdot 15$ & $0 \cdot 82$ \\
\hline rs17465637 & 1q41 (MIA3) & $\mathrm{C}$ & $1 \cdot 14$ & $0 \cdot 75$ \\
\hline rs6725887 & 2q33.1 (WDR12) & $\mathrm{C}$ & $1 \cdot 17$ & $0 \cdot 13$ \\
\hline rs9818870 & $3 q 22.3(M R A S)$ & $\mathrm{T}$ & $1 \cdot 15$ & $0 \cdot 15$ \\
\hline rs17609940 & 6p21.31 (ANKS1A) & G & $1 \cdot 07$ & $0 \cdot 79$ \\
\hline rs9349379 & 6p24.1 (PHACTR1) & G & $1 \cdot 12$ & $0 \cdot 43$ \\
\hline rs12190287 & 6q23.2 (TCF21) & $\mathrm{C}$ & $1 \cdot 08$ & $0 \cdot 63$ \\
\hline rs3798220 & $6 q 25.3(L P A)$ & $\mathrm{C}$ & $1 \cdot 47$ & $0 \cdot 01$ \\
\hline rs10455872 & 6q25.3 (LPA) & G & $1 \cdot 70$ & $0 \cdot 07$ \\
\hline rs11556924 & 7q32.2 (ZC3HC1) & $\mathrm{C}$ & $1 \cdot 09$ & $0 \cdot 64$ \\
\hline rs4977574 & 9p21.3 (CDKN2A) & G & $1 \cdot 29$ & $0 \cdot 55$ \\
\hline rs635634 & $9 q 34.2(A B O)$ & $\mathrm{T}$ & $1 \cdot 10$ & $0 \cdot 21$ \\
\hline rs1746048 & 10q11.21 (CXCL12) & $\mathrm{C}$ & $1 \cdot 17$ & $0 \cdot 86$ \\
\hline rs12413409 & 10q24.32 (CYP17A1) & G & $1 \cdot 12$ & $0 \cdot 90$ \\
\hline rs964184 & 11q23.3 (APOA5) & G & $1 \cdot 13$ & $0 \cdot 13$ \\
\hline rs2259816 & $12 \mathrm{q} 24(H N F 1 A)$ & $\mathrm{T}$ & $1 \cdot 08$ & $0 \cdot 35$ \\
\hline rs3184504 & $12 q 24.12(S H 2 B 3)$ & $\mathrm{T}$ & $1 \cdot 13$ & $0 \cdot 48$ \\
\hline rs4773144 & $13 \mathrm{q} 34(\mathrm{COL} 4 A 1)$ & G & $1 \cdot 07$ & $0 \cdot 41$ \\
\hline rs2895811 & 14q32.2 (HHIPL1) & $\mathrm{C}$ & $1 \cdot 07$ & $0 \cdot 45$ \\
\hline rs3825807 & 15q25.1 (ADAMTS7) & $\mathrm{T}$ & $1 \cdot 08$ & $0 \cdot 57$ \\
\hline rs12936587 & 17p11.2 (RASD1) & G & $1 \cdot 07$ & $0 \cdot 53$ \\
\hline rs216172 & 17p13.3 (SMG6) & $\mathrm{C}$ & $1 \cdot 07$ & $0 \cdot 64$ \\
\hline rs46522 & $17 \mathrm{q} 21.32(U B E 2 Z)$ & $\mathrm{T}$ & $1 \cdot 06$ & $0 \cdot 48$ \\
\hline rs1122608 & $19 \mathrm{p} 13.2(L D L R)$ & G & $1 \cdot 15$ & $0 \cdot 77$ \\
\hline rs9982601 & 21q22.11 (KCNE2) & $\mathrm{T}$ & $1 \cdot 20$ & $0 \cdot 13$ \\
\hline
\end{tabular}

Table 1: 27 SNP panel. Loci information for the SNPS comprising the GRS panel. Data for the SNPS and the risk alleles was obtained from the Malmo diet and cancer study (MDCS) ${ }^{90}$. Position shows chromosomal location and associated gene. 


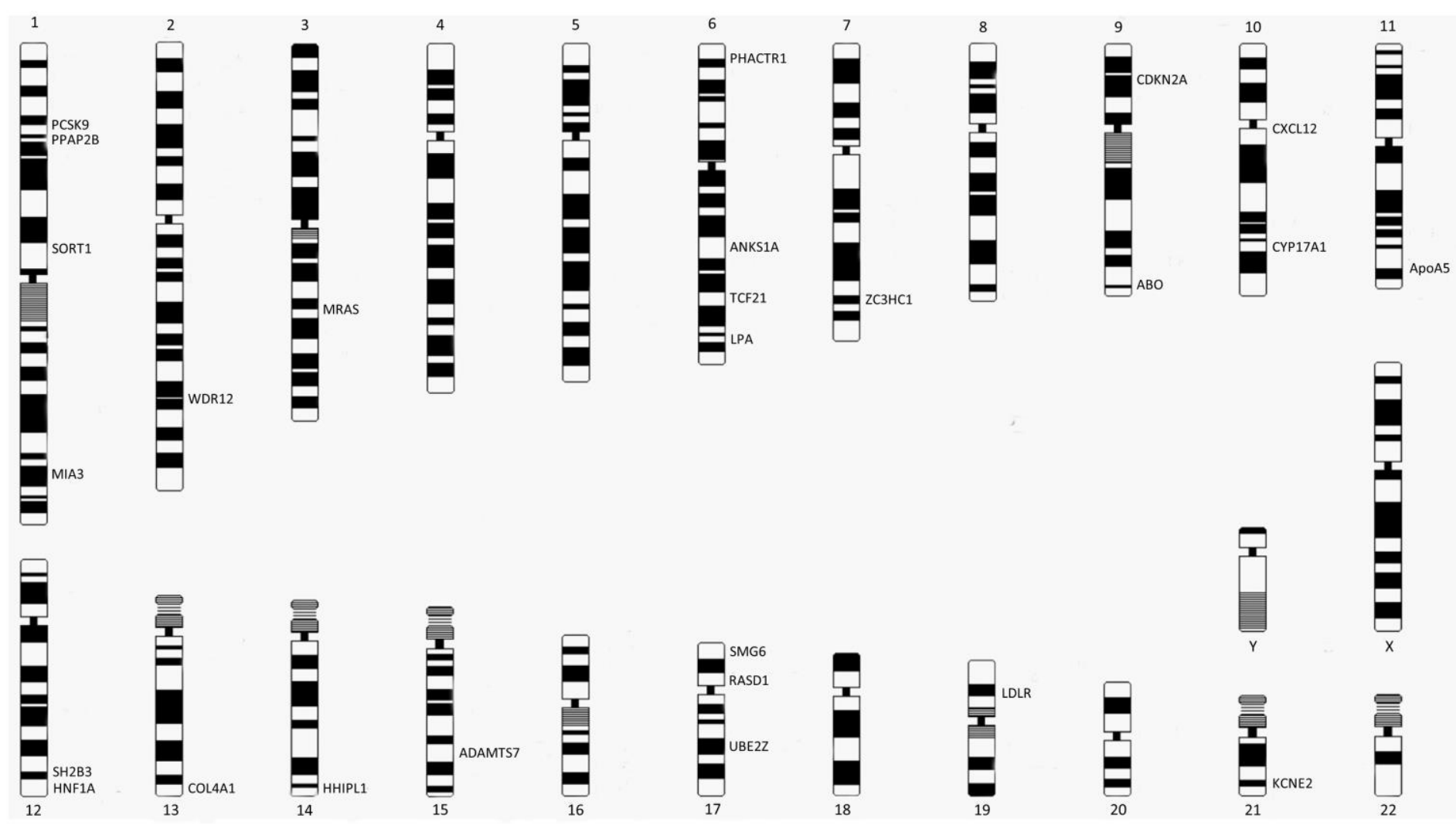

Figure 3: SNP Chromosome ideogram showing location of genes associated with the 27 SNP panel. adapted from Dai et al, 2016 under the Creative Commons Attribution-Noncommercial Licence 


\subsubsection{SNP Panel Gene Functions}

The SNPs that comprise the 27 SNP panel have all been linked to associated genes, as shown in table 1. While some of these genes function within processes that are known to relate to CAD progression, others are in pathways that appear to be novel with regards to CAD. A summary of these genes and their primary functions is below:

\section{SORT1}

The SORT1 gene encodes a membrane glycoprotein named sortilin. Sortilin functions as a sorting receptor, and has been linked to cardiovascular disease in several studies ${ }^{91-93}$. Sortilin that is present in macrophages promotes the uptake of LDL. This may be an important process in the formation of foam cells, and therefore in atherosclerotic lesion development. A deficiency in sortilin may protect against atherosclerosis by reducing LDL uptake from macrophages. An excess of sortilin may exacerbate lesion formation ${ }^{94}$.

\section{$P P A P 2 B$}

PPAP2B codes for phosphatidic acid phosphatase type 2B (PPAP2B). PPAP2B, a type-2 plasma membrane bound protein, catalyzes the dephosphorylation of lysophosphatidic acid (LPA) to form diacylglycerol. It has a role in metabolic pathways controlling the synthesis of glycerophospholipids and triacylglycerols, as well as involvement in receptor-activated signal transduction. Dysregulated LPA has many potential methods of exacerbating atherosclerosis, through inflammatory cytokine release, monocyte adhesion, endothelial integrity changes and LDL uptake ${ }^{95}$.

\section{PCSK 9}

PCSK9 codes for the PCSK9 protein. PCSK9 is a glycoprotein that binds and assists the degradation of LDLR, and thus helps modulate LDL levels. It is highly expressed in the liver, intestine and kidneys. PCSK9 has been proven as a therapeutic target to reduce LDL, often in joint therapy with statins ${ }^{45}$. 
MIA3

The MIA3 gene encodes the melanoma inhibitory activity family member 3 (MIA3) protein, also known as TANGO1 (transport and golgi organisation). MIA3 is located in the ER exit site, where it loads cargo molecules (such as COL7A1) into COPII carriers to promote their secretion out of the ER ${ }^{96}$. MIA3 contributes to both tumour suppression ${ }^{97}$ and progression ${ }^{98}$.

\section{WDR12}

The WDR12 gene codes for the WD repeat domain 12 protein (WDR12). WDR12 is a member of the WD repeat protein family. The members of this family are involved in many cellular processes, including cell cycle progression, signal transduction, apoptosis and gene regulation. WDR12 Participates in ribosome biogenesis and cell proliferation $^{99}$. Deletion of WDR12 inhibits cell proliferation ${ }^{100}$. Over expression of WDR12 could deteriorate both systolic and diastolic function of the rat heart. Also, a WDR12 variant was associated with diastolic dysfunction in human subjects ${ }^{101}$.

\section{MRAS}

M-ras, encoded by the MRAS gene is a ras-related protein. It is a membrane anchored, intracellular signal transducer. MRAS is activated in a significant number of tumours and is therefore implicated in many types of cancer ${ }^{102}$. The M-ras protein is highly expressed in the cardiovascular system and is thought to have a role in adhesion signaling ${ }^{103}$.

\section{ANKS1A}

ANKS1A codes for Ankyrin Repeat and SAM domain-containing protein $1 \mathrm{~A}$ which is also known as Odin. Odin is an effector of EGFR recycling ${ }^{104}$.

\section{PHACTR1}

Phosphatase and Actin Regulator 1 (PHACTR1) controls PP1 activity and F-actin remodelling. It is involved in the control of tubulogenesis, actin polymerization and lamellipodial dynamics ${ }^{105}$. PHACTR1 is thought to play a role in cell motility and vascular morphogenesis ${ }^{106}$. Suppression of PHACTR1 leads to increased expression of death cell receptors, leading to apoptosis ${ }^{107}$. PHACTR1 is often found in GWAS studies 
for coronary artery disease, however little is known about PHACTR1 function within the heart. In muscle cells however, it can bind actin and regulate the reorganization of the actin cytoskeleton.

\section{TCF21}

Transcription Factor 21 (TCF21) manages cell fate specification, commitment and differentiation in a number of cell lineages. It is involved in the specification of the cardiac fibroblast lineage ${ }^{108}$. Without TCF21, fibroblast progenitor cells do not undergo epithelial-to-mesenchymal transition. TCF21 has structural heart effects and is involved in coronary artery integrity. TCF21 is a tumour suppressor which is silenced in many cancers ${ }^{109}$.

\section{$L P A$}

LPA codes for Lipoprotein (a) [Lp(a)]. Lp(a) consists of an LDL-like particle and apolipoprotein (a) [apo(a)], which is covalently bound to the apoB moiety of the LDLlike particle. Plasma concentration of $\operatorname{Lp}(\mathrm{a})$ is highly heritable and mainly controlled by the LPA gene. The structure of $\mathrm{Lp}(\mathrm{a})$ is largely similar to plasminogen and tPA and it competes with plasminogen for its binding site, leading to reduced fibrinolysis. Also, Lp(a) stimulates secretion of PAI-1, which can lead to thrombogenesis. Lp(a) carries cholesterol, thus contributes to atherosclerosis ${ }^{110}$. $\mathrm{Lp}(\mathrm{a})$ transports oxidized phospholipids, which attract inflammatory cells to vessel walls and leads to smooth muscle cell proliferation ${ }^{111-113}$.

\section{ZC3HC1}

Zinc Finger, C3HC-type containing 1 is also known as nuclear interacting partner of ALK (NIPA). The rs11556924 SNP results in an arginine to histidine polymorphism at amino acid residue 363 in ZC3HC1. ZC3HC1 is broadly expressed, especially in the heart, skeletal muscles and testis ${ }^{114}$. ZC3HC1 is involved in cell cycle regulation ${ }^{115,116}$.

\section{CDKN2A}

Cyclin dependent kinase inhibitor 2A (CDKN2A) is associated with many diseases, including $\mathrm{CAD}$, diabetes and many cancers ${ }^{117}$. It is involved in transcriptional 
repression. The gene codes for 3 proteins (INK4A, ARF, INK4B) that act as tumour suppressors by regulating the cell cycle ${ }^{118}$.

\section{$A B O$}

The $A B O$ gene encodes the histo-blood group $\mathrm{ABO}$ system transferase. This gene determines the $\mathrm{ABO}$ blood group of an individual. This is achieved through the modification of oligosaccharides on cell surface glycoproteins. Variations in the sequence of the protein determine the type of modification and therefore the blood group of the individual119,120. Blood group differences could be linked to MI as the different groups have varying levels of clotting factors ${ }^{121}$.

\section{CXCL12}

C-X-C motif Chemokine 12 otherwise known as Stromal Cell Derived Factor 1 (SDF1) is a chemokine protein. It is a member of the intercrine family and is involved in embryogenesis, immune surveillance, inflammation response and tissue homeostasis as well as tumour growth and metastasis ${ }^{122}$. CXCL12 is a chemoattractant active on Tlymphocytes and monocytes. It activates CXCR4 to induce a rapid, transient rise in the level of intracellular $\mathrm{Ca}^{2+}$. CXCL12 is a regulator of monocyte migration and adhesion and has been implicated in heart disease ${ }^{123,124}$. CXCL12 has been proposed as a method of stem cell based tissue regeneration following cardiac myopathy ${ }^{125,126 .}$

\section{CYP17A1}

Cytochrome P450 17A1 (CYP17A1) is a monooxygenase that catalyzes reactions in drug metabolism as well as synthesis of cholesterol, steroids and other lipids. The CYP17A1 enzyme catalyzes a carbon-carbon bond scission among other functions ${ }^{127}$.

\section{APOA5}

Apolipoprotein A V (APOA5) is a regulator of plasma triglyceride levels and a component of VLDL and HDL. It is hypothesized that APOA5 affects lipoprotein metabolism by interacting with the LDL-R gene family receptors ${ }^{128}$. 
HNF1A

HNF1 homeobox A (HNF1A) is a transcription factor that is highly expressed in the liver. It regulates expression of a number of liver specific genes ${ }^{129}$. HNF1A also has possible links to levels of C-reactive protein ${ }^{130}$.

\section{SH2B3}

SH2B adapter protein 3 (SH2B3) is a regulator of signaling pathways in hematopoiesis, inflammation and cell migration ${ }^{131}$. It is involved in a wide range of diseases, including blood diseases, autoimmune disorders and vascular disease ${ }^{132}$.

\section{COL4A1}

Collagen, Type IV, Alpha 1 (COL4A1) is a subunit of type IV collagen. Type IV collagen is the major structural component of basement membranes. In a normal artery wall, collagen type IV inhibits smooth muscle cell proliferation ${ }^{133}$.

\section{HHIPL1}

Hedgehog Interacting Protein (HHIPL1) is expressed in arterial endothelial cells. It is involved in vascular remodeling and is downregulated in tumour cells ${ }^{134}$. HHIPL1 is abundant in aortic endothelial cells and affects vascular remodelling through regulation of the Hedgehog-VEGF-Notch signaling cascade ${ }^{135}$.

\section{ADAMTS7}

A disintegrin and metalloproteinase with thrombospondin motifs 7 (ADAMTS7) is a zinc dependent protease that catalyzes the degradation of cartilage oligomeric matrix protein (COMP) ${ }^{136}$ and mediates VSMC migration ${ }^{137}$. A deficiency in ADAMTS7 in hyperlipidemic mice attenuates the formation of atherosclerotic lesions, therefore inhibition of ADAMTS7 could be atheroprotective ${ }^{138}$.

\section{RASD1}

Dexamethasone-induced Ras-related protein 1 (RASD1) is a member of the Ras superfamily of small G-proteins. RASD1 regulates signal transduction pathways through G-proteins and GPCRs and in this way can prevent aberrant cell growth ${ }^{139}$. 
SMG6

SMG6 codes for the protein telomerase-binding protein EST1A. SMG6 binds single stranded DNA in telomere maintenance and ssRNA in nonsense-mediated mRNA decay 140,141. SMG6 functions in both telomere maintenance and RNA surveillance pathways ${ }^{140}$.

$U B E 2 Z$

Ubiquitin conjugating enzyme E2 Z (UBE2Z) is an E2 ubiquitin conjugating enzyme that is involved in protein ubiquination, which is a major component of protein degradation, during proteolysis ${ }^{142}$.

$L D L R$

The low-density lipoprotein receptor $(L D L R)$ gene family are cell surface proteins that are involved in receptor mediated endocytosis of specific ligands. LDL is normally bound at the cell membrane and taken into the cell, ending up in lysosomes where it can be degraded and the cholesterol made available for HMG CoA reductase in the cholesterol synthesis pathway. Mutations in $L D L R$ can lead to familial hypercholesterolemia (FH) and premature MI143.

KCNE2

Potassium voltage-gated channel subfamily E member 2 (KCNE2) is a voltage-gated potassium channel subunit that is associated with long QT syndrome ${ }^{144}$. KCNE2 has the ability to regulate a number of different ion channels, and therefore influences multiple cell types and tissues ${ }^{144,145}$. 


\subsection{Thesis Aims}

This study aims to investigate the variation in genetic risk of ACS, as measured by a GRS in a New Zealand based population.

Our primary aim is to assess the differences in GRS values between a premature MI patient cohort (under 50 years old) and a 60 years or older control group.

We will also analyze variation in GRS values between patients of different ethnicities (Māori and non-Māori), and evaluate associations between the GRS and traditional risk factors including hypertension, dyslipidaemia, diabetes and obesity, as well as family history of CAD and gender.

This study will also enable us to investigate the distribution of the GRS values in the NZ population, and to compare the risk allele frequencies of the individual SNPs between the study groups (premature MI vs. over 60 MI, Māori vs. non-Māori, and Total NZ study population vs. reported overseas based populations).

These questions will enable us to gain further understanding of the utility of genetic risk scoring in ACS risk prediction. We will also be able to assess whether this specific GRS, developed by Mega et al, has the ability to determine genetic risk for ACS in a New Zealand based population. 


\section{Chapter 2 - Methods:}

\section{1 - Study Population}

We screened patients who presented to Wellington Regional Hospital with an ACS between January 2012 and March 2016. Patients were required to have a coronary angiogram planned ( \pm PCI) and must have been adequately pre-treated with dual antiplatelet therapy (DAPT). DAPT was defined as chronic therapy with aspirin $(\geq 75$ $\mathrm{mg} /$ day) and either Clopidogrel ( $\geq 75 \mathrm{mg} /$ day) or Ticagrelor ( $\geq 90 \mathrm{mg} /$ day) and/or loading with aspirin $\geq 300 \mathrm{mg}$ and Clopidogrel or Ticagrelor $\geq 300 \mathrm{mg}$ at least 6 hours prior to enrolment. Criteria for the diagnosis of an ACS included symptoms suggestive of myocardial ischemia lasting longer than 10 minutes, combined with elevated troponin levels and/or $\geq 1 \mathrm{~mm}$ of new ST segment deviation or T wave inversion on an electrocardiogram (ECG) in at least 2 contiguous leads, as fitting the Third Universal Definition of Myocardial Infarction ${ }^{34}$. Exclusion criteria included a platelet count of less than $100 \times 10^{9} / \mathrm{L}$, a known platelet function disorder, administration of a thrombolytic agent within 24 hours of enrolment or administration of an oral antiplatelet agent other than Aspirin, Clopidogrel or Ticagrelor within a week prior to enrolment. This study was reviewed and approved by the Lower South Ethics Committee (LRS/11/09/035). Participation in this study was voluntary, and each patient gave informed written consent at the time of recruitment.

From within our biobank we identified 140 patients for our premature myocardial infarction cohort based upon age (under 50 years), and the presence of verified coronary artery disease, classified as observation of an occlusion of greater than $50 \%$ being found during angiography. Our control population $(n=280)$ was selected by filtering for patients who experienced their first MI at 60 years or older, with verified obstructive disease (greater than $50 \%$ occlusion). 


\section{2 - Data Collection}

Patient data including demographics, clinical characteristics, previous medical history, medications, full blood count, cardiac enzyme profile and observations at time of admission were recorded prospectively from patient medical records at the time of enrolment. Procedural variables and in hospital outcomes were noted.

\section{3 - Blood Collection and Storage}

Blood samples were collected from the patient as they underwent angiography in the Catheterization Laboratory. Whole blood samples were stored in EDTA treated blood collection tubes and stored at $-80^{\circ} \mathrm{C}$ within our Wellington Acute Coronary Syndrome registry blood bank in our laboratory at Wellington Hospital.

\section{4 - DNA Extraction}

DNA was extracted from $3 \mathrm{ml}$ EDTA treated whole blood samples using the Promega Wizard ${ }^{\circledR}$ Genomic DNA Purification Kit as per manufacturer guidelines (Promega Corporation, Madison, WI).

$3 \mathrm{ml}$ of whole blood was added to $9 \mathrm{ml}$ of cell lysis solution in $15 \mathrm{ml}$ falcon tubes.

The tubes were capped and placed on the suspension mixer for 12 minutes and were then centrifuged at $5000 \mathrm{rpm}$ for 5 minutes.

We then ensured the white cell pellet was at the base of the tube, before we poured off the supernatant, making sure the cell pellet did not dislodge.

The pellet was gently washed with $3 \mathrm{ml}$ of $0.9 \%$ Sodium Chloride before the tube was inverted to dry.

$3 \mathrm{ml}$ of nuclei lysis solution was then added to the pellet, and the white blood cells were resuspended using a Pasteur pipette.

We added $1 \mathrm{ml}$ of protein precipitation solution, replaced the cap on the tube and vortexed for 20 seconds.

The sample was centrifuged at $5000 \mathrm{rpm}$ for 5 minutes once more before the supernatant was transferred to a clean second tube.

$3 \mathrm{ml}$ of isopropanol was added to the supernatant in the second tube and the sample was placed on the suspension mixer for one minute to precipitate the DNA.

The sample was centrifuged for one minute at $5000 \mathrm{rpm}$, then the supernatant was decanted, being sure to retain the DNA pellet. 
$3 \mathrm{ml}$ of $70 \%$ ethanol was added to the sample and mixed gently by inversion.

The sample was centrifuged for one minute at $5000 \mathrm{rpm}$, then the supernatant was decanted, retaining the DNA pellet.

The tubes were inverted to drain for approximately 10 minutes before being placed inverted in a $37^{\circ} \mathrm{C}$ incubator for one hour.

$200 \mu \mathrm{l}$ of DNA rehydration solution (TE buffer) was added and then the capped tubes were returned to the $37^{\circ} \mathrm{C}$ incubator overnight.

The $200 \mu \mathrm{l}$ of rehydrated DNA solution was then transferred into $0.65 \mathrm{~mL}$ Eppendorf tubes and stored at $4^{\circ} \mathrm{C}$.

The concentration of genomic DNA was measured using the Qubit Fluorometer (Thermo Fisher Scientific Inc, Waltham, MA). The samples were then standardized to 10 $\mathrm{ng} / \mu \mathrm{l}$. 


\section{5 - Genotyping}

Genotyping was performed on the Sequenom MassARRAY iPLEX Platform following PCR amplification of the SNP regions ${ }^{146}$.

DNA samples at $10 \mathrm{ng} / \mathrm{ul} \mathrm{PCR}$

- Make a master mix consisting of the primers for all 27 loci + buffer $+\mathrm{MgCl}^{2}+\mathrm{dNTPs}$ + water + enzyme

- Add $3 \mu \mathrm{l}$ of master mix $+2 \mu \mathrm{l}$ of sample per well in a 96 well plate

- PCR thermocycling conditions:

○ 94 degrees - 2 minutes

- 94 degrees - 30 seconds

○ 56 degrees -30 seconds | 45 cycles

○ 72 degrees - 1 minute

○ 72 degrees - 5 minutes

○ 4 degrees - hold

- Shrimp Alkaline Phosphatase (SAP) treatment:

- Deactivates remaining dNTPs

- 37 degrees 40 minutes

- 85 degrees 5 minutes

- 4 degrees hold

○ Heat deactivated

- Extension

- Add Terminator mix of ddNTPs to extend SNP (single base extension)

○ ddNTPs are weighted (only 2 of them) to increase the differences between the weights so that it can be more accurately measured by the MALDI-TOF.

$\circ$ Extension reaction thermocycling conditions

- 94 degrees 30 seconds

- 94 degrees 5 seconds

- 52 degrees 5 seconds | 5 cycles | 40 cycles

- 80 degrees 5 seconds

- 72 degrees 3 minutes

- 4 degrees hold 
○ No-Template Controls were included (water)

- Final volume from the PCR stage is $9 \mu \mathrm{l}$

○ $16 \mu \mathrm{l}$ of water is added to dilute the sample to the concentration required by the Sequenom ( $25 \mu$ l total volume in well)

- Also, add resin at this step to de-ionize/de-salt solution

- Rotate slowly for 30 minutes

- Centrifuge after so that the resin sits at the bottom of the wells

- Ensure that the primers are balanced for the mass spec

Set up the assay on the Sequenom software:

- $\quad$ Set up CAD panel

- Select points on the plate to take from and points on the chip to spot on to

- Input sample IDs into software - correlate with location on the plate and where being spotted on the chip

- The Nanodispenser will spot on the chip

- Machine tips protocol:

- Rinse in water

- Sonicate in $50 \%$ ethanol

- Dry

- Pick up sample

- Spot

- Repeat

- Once the samples have been loaded onto the chip, load the chip into the Sequenom MALDITOF

- Once the required level of vacuum has been reached, 10 shots will be fired at the sample, leading to excitation and flight
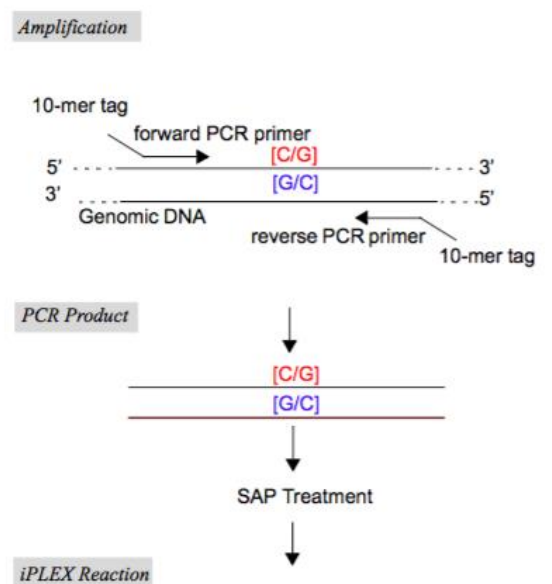

Sample conditioning, dispensing, and MALDI-TOF MS

Spectrum

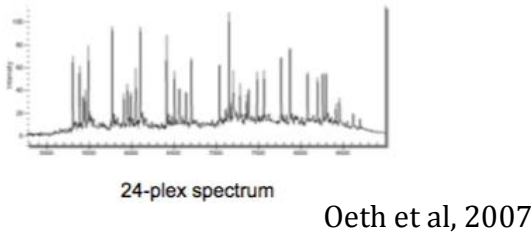

Figure 4: iPLEX single assay process

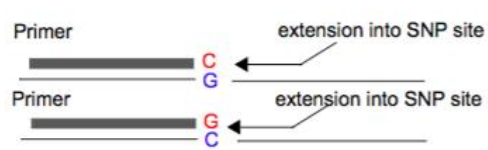


- Data output with spectra can be manually checked - check alignment and calls i.e. rule out adducts, clarify peaks, yield vs spread etc.

- Once calls have been adjudicated a genotyping report can be exported in .xls format ${ }^{147}$. 


\section{6 - Construction of the Genetic Risk Score}

The genetic risk score was calculated from the presence or absence of the 27 SNPs in the panel. The number of risk alleles present $(0,1$ or 2$)$ was weighted by the log of the odds ratio for each of the SNPs. A linear sum of these then produced the genetic risk score for each patient.

This took the form of:

$$
\sum(a \cdot \log O R)
$$

Where:

$a=$ number of risk alleles present per SNP

$O R=$ odds ratio for each SNP

For example, a SNP with an odds ratio of 1.19 with one risk allele present would be calculated as:

$1 \times \log 1.19$

$\log 1.19=0.075546961$

$0.075546961 \times 1=0.075546961$ 
This calculation is repeated for each of the 27 SNPs for every patient in the study. Creating a list of values such as this:

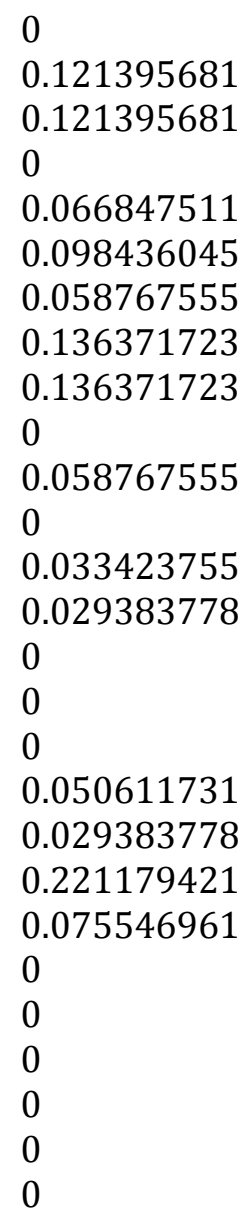

These values are then summed to give the GRS for that patient.

In this example GRS = 1.237882899

\section{7 - Statistical Analysis}

Risk scores and other continuous variables were stated as means with standard deviations and were compared using Student's t-tests. Categorical data was reported as counts with percentage proportions and compared using Pearson's chi-squared tests. Statistical analysis was carried out in IBM SPSS Statistics 23 (IBM Corporation, Armonk, NY). Graphing was carried out in GraphPad Prism 7 (GraphPad Software Inc, La Jolla, CA) and Microsoft Excel 2013 (Microsoft Corporation, Redmond, WA). 
Hadley Northcott 
Chapter 3 - Results

\subsection{Patient Demographics}

\begin{tabular}{|c|c|c|c|c|}
\hline Demographics, n (\%) & $\begin{array}{l}\text { Total } \\
n=420\end{array}$ & $\begin{array}{l}\text { Premature } \\
\text { ACS <50yr } \\
n=140\end{array}$ & $\begin{array}{l}\text { ACS } \geq 60 y r s \\
n=280\end{array}$ & P-value \\
\hline Male & $302(71.9)$ & $113(80.7)$ & $189(67.5)$ & $0.005^{\mathrm{a}}$ \\
\hline Age (mean \pm SD) & $60.9 \pm 13.2$ & $44.6 \pm 4.1$ & $69.1 \pm 7.1$ & $<0.0001^{b}$ \\
\hline BMI $($ mean \pm SD $)$ & $29.1 \pm 5.8$ & $30.8 \pm 6.2$ & $28.3 \pm 5.4$ & $<0.0001^{b}$ \\
\hline \multicolumn{5}{|l|}{ Ethnicity } \\
\hline European & $353(84.0)$ & $100(71.4)$ & $253(90.4)$ & \\
\hline Māori & $40(9.5)$ & $26(18.6)$ & $14(5)$ & \\
\hline Pacific Islander & $11(2.6)$ & $5(3.6)$ & $6(2.1)$ & $<0.0001^{\mathrm{a}}$ \\
\hline Other & $16(3.8)$ & $9(6.4)$ & $7(2.5)$ & \\
\hline \multicolumn{5}{|l|}{ Risk Factors, n (\%) } \\
\hline Hypertension & $235(56.0)$ & $52(37.1)$ & $183(65.4)$ & $<0.0001^{\mathrm{a}}$ \\
\hline Family History & $158(37.6)$ & $64(45.7)$ & $94(33.6)$ & $0.015^{\mathrm{a}}$ \\
\hline Dyslipidemia & $264(62.9)$ & $78(55.7)$ & $186(66.4)$ & $0.032^{\mathrm{a}}$ \\
\hline Diabetes & $74(17.6)$ & $19(13.6)$ & $55(19.6)$ & $0.124^{\mathrm{a}}$ \\
\hline Obese & $149(35.5)$ & $70(50)$ & $79(28.2)$ & $<0.0001^{\mathrm{a}}$ \\
\hline \multicolumn{5}{|l|}{ Smoking } \\
\hline Current & $103(24.5)$ & $65(46.4)$ & $38(13.6)$ & \\
\hline Former & $164(39.0)$ & $34(24.3)$ & $130(46.4)$ & $<0.0001^{\mathrm{a}}$ \\
\hline Never & $153(36.4)$ & $41(29.3)$ & $112(40.0)$ & \\
\hline \multicolumn{5}{|c|}{$\begin{array}{l}\text { Clinical Presentation, } n \\
\text { (\%) }\end{array}$} \\
\hline STEMI & $93(22.1)$ & $29(20.7)$ & $64(22.9)$ & \\
\hline NSTEMI & 327 (77.9) & $111(79.3)$ & $216(77.1)$ & $0.618^{a}$ \\
\hline
\end{tabular}

Table 2: Clinical characteristics of the study population.

${ }^{a}=$ Chi-Square test,${ }^{b}=$ Student's $t$-test. $S D=$ Standard deviation. 
420 patients met the inclusion criteria for this study, their clinical characteristics and baseline demographic data is shown in table 2. They were $71.9 \%$ male, $9.5 \%$ were of Māori ethnicity and 37.6\% had a family history of CAD. Our premature MI cohort had an average age of 44.6, a mean BMI of 30.8 and were comprised of 18.6\% Māori. They had significantly more males and patients of Māori and Pacific Island ethnicity than the over 60 year olds control cohort and also had a higher average BMI. The premature MI cohort were less likely to have hypertension or dyslipidemia and more likely to be current smokers. A family history of ACS was most prevalent in the premature MI cohort, at $45.7 \%$ compared to $33.6 \%$ in the older group.

\subsection{SNP frequencies}

\subsubsection{Between Premature MI and Over $60 \mathrm{MI}$}

\section{Risk Allele Frequency}

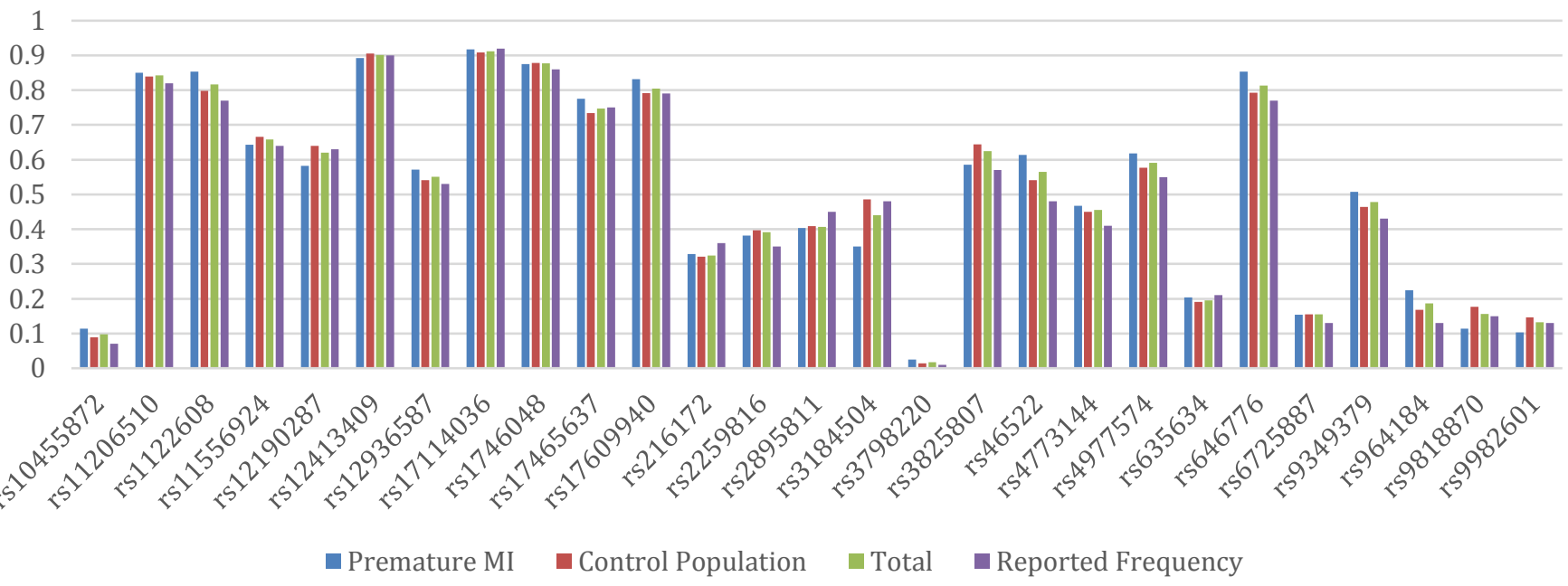

Figure 5: Risk allele SNP frequencies between study groups. Reported SNP frequency data is from the Malmo Diet and Cancer Study ${ }^{90}$. 


\begin{tabular}{|c|c|c|c|c|c|c|c|c|c|c|}
\hline & & & & Control & & & & & & \\
\hline & Premature & & & Populatio & & & Total & & & Reported \\
\hline$\underline{\text { SNP }}$ & $\underline{\text { MI }}$ & $\underline{\text { CI }}$ & - & $\underline{\mathbf{n}}$ & $\underline{\text { CI }}$ & - & Population & $\underline{\text { CI }}$ & - & Frequency \\
\hline rs10455872 & 0.11 & 0.075 & 0.154 & 0.09 & 0.065 & 0.114 & 0.10 & 0.076 & 0.119 & 0.07 \\
\hline rs11206510 & 0.85 & 0.742 & 0.958 & 0.84 & 0.763 & 0.915 & 0.84 & 0.781 & 0.905 & 0.82 \\
\hline rs1122608 & 0.85 & 0.745 & 0.962 & 0.80 & 0.724 & 0.872 & 0.82 & 0.756 & 0.878 & 0.77 \\
\hline rs11556924 & 0.64 & 0.549 & 0.737 & 0.67 & 0.598 & 0.734 & 0.66 & 0.603 & 0.713 & 0.64 \\
\hline rs12190287 & 0.58 & 0.493 & 0.672 & 0.64 & 0.573 & 0.706 & 0.62 & 0.567 & 0.673 & 0.63 \\
\hline rs12413409 & 0.89 & 0.782 & 1.000 & 0.91 & 0.827 & 0.984 & 0.90 & 0.837 & 0.965 & 0.90 \\
\hline rs12936587 & 0.57 & 0.483 & 0.660 & 0.54 & 0.480 & 0.602 & 0.55 & 0.501 & 0.601 & 0.53 \\
\hline rs17114036 & 0.92 & 0.806 & 1.000 & 0.91 & 0.830 & 0.988 & 0.91 & 0.847 & 0.976 & 0.92 \\
\hline rs1746048 & 0.88 & 0.765 & 0.985 & 0.88 & 0.801 & 0.956 & 0.88 & 0.814 & 0.941 & 0.86 \\
\hline rs17465637 & 0.78 & 0.672 & 0.878 & 0.73 & 0.663 & 0.805 & 0.75 & 0.689 & 0.806 & 0.75 \\
\hline rs17609940 & 0.83 & 0.725 & 0.939 & 0.79 & 0.717 & 0.865 & 0.80 & 0.744 & 0.865 & 0.79 \\
\hline rs216172 & 0.33 & 0.261 & 0.396 & 0.32 & 0.274 & 0.368 & 0.32 & 0.285 & 0.362 & 0.36 \\
\hline rs2259816 & 0.38 & 0.310 & 0.455 & 0.40 & 0.344 & 0.449 & 0.39 & 0.349 & 0.434 & 0.35 \\
\hline rs2895811 & 0.40 & 0.329 & 0.478 & 0.41 & 0.356 & 0.462 & 0.41 & 0.364 & 0.450 & 0.45 \\
\hline rs3184504 & 0.35 & 0.281 & 0.419 & 0.49 & 0.428 & 0.543 & 0.44 & 0.396 & 0.485 & 0.48 \\
\hline rs3798220 & 0.03 & 0.006 & 0.044 & 0.01 & 0.004 & 0.024 & 0.02 & 0.009 & 0.027 & 0.01 \\
\hline rs3825807 & 0.59 & 0.496 & 0.675 & 0.64 & 0.578 & 0.711 & 0.63 & 0.572 & 0.678 & 0.57 \\
\hline rs46522 & 0.61 & 0.522 & 0.706 & 0.54 & 0.480 & 0.602 & 0.57 & 0.515 & 0.616 & 0.48 \\
\hline rs4773144 & 0.47 & 0.388 & 0.548 & 0.45 & 0.394 & 0.506 & 0.46 & 0.410 & 0.502 & 0.41 \\
\hline rs4977574 & 0.62 & 0.526 & 0.710 & 0.58 & 0.514 & 0.640 & 0.59 & 0.539 & 0.642 & 0.55 \\
\hline rs635634 & 0.20 & 0.151 & 0.256 & 0.19 & 0.155 & 0.227 & 0.20 & 0.165 & 0.225 & 0.21 \\
\hline rs646776 & 0.85 & 0.745 & 0.962 & 0.79 & 0.719 & 0.867 & 0.81 & 0.752 & 0.874 & 0.77 \\
\hline rs6725887 & 0.15 & 0.108 & 0.199 & 0.16 & 0.123 & 0.188 & 0.15 & 0.128 & 0.181 & 0.13 \\
\hline rs9349379 & 0.51 & 0.424 & 0.591 & 0.46 & 0.408 & 0.521 & 0.48 & 0.432 & 0.525 & 0.43 \\
\hline rs964184 & 0.23 & 0.169 & 0.281 & 0.17 & 0.134 & 0.202 & 0.19 & 0.158 & 0.216 & 0.13 \\
\hline rs9818870 & 0.11 & 0.075 & 0.154 & 0.18 & 0.142 & 0.212 & 0.16 & 0.129 & 0.183 & 0.15 \\
\hline rs9982601 & 0.10 & 0.066 & 0.141 & 0.15 & 0.115 & 0.178 & 0.13 & 0.108 & 0.157 & 0.13 \\
\hline
\end{tabular}

Table 3: Risk allele SNP frequencies between study groups. Reported SNP frequency data is from the Malmo Diet and Cancer Study ${ }^{90} . C I=95 \%$ Confidence Interval, MI = Myocardial Infarction, SNP = Single Nucleotide Polymorphism. Red highlighting indicates SNPS with significant differences in frequencies. 
Using 95\% confidence intervals, we saw that there were four SNPs that were present in statistically significant different frequencies between our total study population and the reported frequencies from the Malmo Diet and Cancer Study (MDCS). These SNPs were rs10455872, rs46522, rs9349379 and rs964184 which are associated with the genes LPA, UBE2Z, PHACTR1 and APOA5 respectively. All four SNPs were present at higher frequencies within our CAD population. The remaining 23 SNPs were present at similar levels between the two populations.

We observed one difference in risk allele frequency between the premature MI and control population that was significant. This was the rs3184504 SNP which is associated with the SH2B3 gene. All other risk alleles were present at similar levels between the age group study populations. SNP frequency was calculated as the number of risk alleles present as a percentage of the total number of alleles in the population.

\subsubsection{Between Māori and non-Māori}

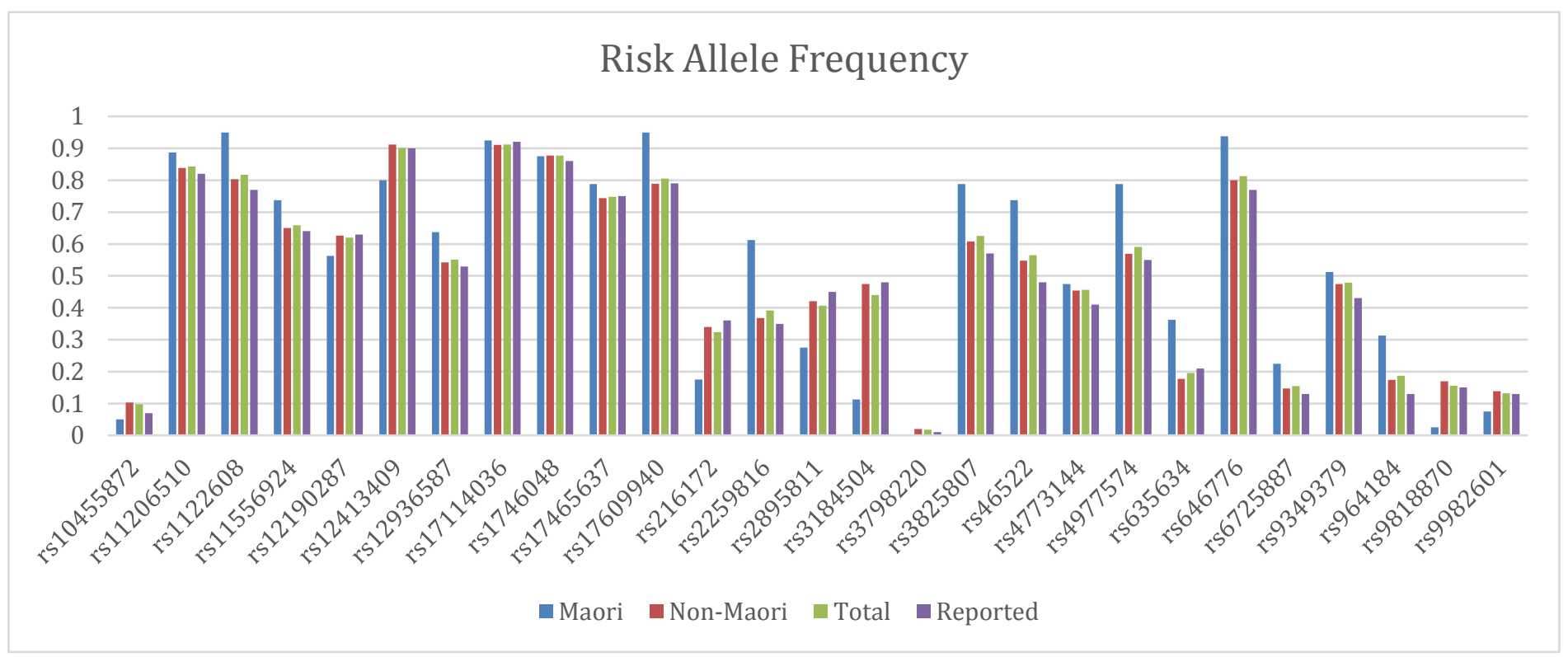

Figure 6: Risk allele SNP frequencies in Maori and non-Maori. Reported SNP frequency data is from the Malmo Diet and Cancer Study 90.

Six SNPs were present in significantly different frequencies between the Māori and nonMāori populations. These changes in frequency include both more abundant and less abundant rates of certain SNPs in the Māori population. Although more variation appears to be present when looking at the graph, using 95\% confidence intervals, we can only see 
significance in six of these. This is most likely due to the size of the study population, a larger cohort would likely reduce the size of the confidence intervals, allowing us to be more confident in differences observed between the study populations. The six SNPs that were significantly different are; rs216172, rs2259816, rs3184504, rs3798220, rs635634 and rs9818870. The differences in allele frequency at the rs3798220 and rs9818870 loci are most likely due to the very low overall frequency of these SNPs. The risk alleles for rs2259816 and rs635634 were more frequent within the Māori cohort and the risk alleles for rs216172 and rs3184504 were less frequent within the Māori cohort. 


\begin{tabular}{|c|c|c|c|c|c|c|c|c|c|}
\hline \multirow{3}{*}{$\begin{array}{l}\text { SNP } \\
r s 10455872\end{array}$} & \multirow{3}{*}{$\begin{array}{l}\text { Maori } \\
0.05\end{array}$} & \multirow[b]{2}{*}{$\underline{\text { CI }}$} & & \multicolumn{5}{|l|}{ Non- } & \multirow[b]{3}{*}{0.119} \\
\hline & & & & $\underline{\text { Maori }}$ & $\underline{\text { CI }}$ & & Total & $\underline{\text { CI }}$ & \\
\hline & & 0.001 & 0.099 & 0.10 & 0.080 & 0.125 & 0.10 & 0.076 & \\
\hline rs11206510 & 0.89 & 0.681 & 1.000 & 0.84 & 0.773 & 0.903 & 0.84 & 0.781 & 0.905 \\
\hline rs1122608 & 0.95 & 0.736 & 1.000 & 0.80 & 0.739 & 0.866 & 0.82 & 0.756 & 0.878 \\
\hline rs11556924 & 0.74 & 0.549 & 0.926 & 0.65 & 0.593 & 0.707 & 0.66 & 0.603 & 0.713 \\
\hline rs12190287 & 0.56 & 0.398 & 0.727 & 0.63 & 0.570 & 0.683 & 0.62 & 0.567 & 0.673 \\
\hline rs12413409 & 0.80 & 0.604 & 0.996 & 0.91 & 0.844 & 0.980 & 0.90 & 0.837 & 0.965 \\
\hline rs12936587 & 0.64 & 0.463 & 0.812 & 0.54 & 0.490 & 0.594 & 0.55 & 0.501 & 0.601 \\
\hline rs17114036 & 0.93 & 0.714 & 1.000 & 0.91 & 0.843 & 0.978 & 0.91 & 0.847 & 0.976 \\
\hline rs1746048 & 0.88 & 0.670 & 1.000 & 0.88 & 0.811 & 0.944 & 0.88 & 0.814 & 0.941 \\
\hline rs17465637 & 0.79 & 0.593 & 0.982 & 0.74 & 0.682 & 0.805 & 0.75 & 0.689 & 0.806 \\
\hline rs17609940 & 0.95 & 0.736 & 1.000 & 0.79 & 0.726 & 0.853 & 0.80 & 0.744 & 0.865 \\
\hline rs216172 & 0.18 & 0.083 & 0.267 & 0.34 & 0.298 & 0.381 & 0.32 & 0.285 & 0.362 \\
\hline rs2259816 & 0.61 & 0.441 & 0.784 & 0.37 & 0.325 & 0.412 & 0.39 & 0.349 & 0.434 \\
\hline rs2895811 & 0.28 & 0.160 & 0.390 & 0.42 & 0.375 & 0.467 & 0.41 & 0.364 & 0.450 \\
\hline rs3184504 & 0.11 & 0.039 & 0.186 & 0.48 & 0.426 & 0.524 & 0.44 & 0.396 & 0.485 \\
\hline rs3798220 & 0.00 & 0.000 & 0.000 & 0.02 & 0.010 & 0.030 & 0.02 & 0.009 & 0.027 \\
\hline rs3825807 & 0.79 & 0.593 & 0.982 & 0.61 & 0.552 & 0.663 & 0.63 & 0.572 & 0.678 \\
\hline$r s 46522$ & 0.74 & 0.549 & 0.926 & 0.55 & 0.495 & 0.600 & 0.57 & 0.515 & 0.616 \\
\hline rs4773144 & 0.48 & 0.324 & 0.626 & 0.45 & 0.406 & 0.502 & 0.46 & 0.410 & 0.502 \\
\hline rs4977574 & 0.79 & 0.593 & 0.982 & 0.57 & 0.516 & 0.623 & 0.59 & 0.539 & 0.642 \\
\hline rs635634 & 0.36 & 0.231 & 0.494 & 0.18 & 0.148 & 0.208 & 0.20 & 0.165 & 0.225 \\
\hline rs646776 & 0.94 & 0.725 & 1.000 & 0.80 & 0.736 & 0.864 & 0.81 & 0.752 & 0.874 \\
\hline rs6725887 & 0.23 & 0.121 & 0.329 & 0.15 & 0.120 & 0.175 & 0.15 & 0.128 & 0.181 \\
\hline rs9349379 & 0.51 & 0.356 & 0.669 & 0.48 & 0.426 & 0.524 & 0.48 & 0.432 & 0.525 \\
\hline rs964184 & 0.31 & 0.190 & 0.435 & 0.17 & 0.144 & 0.203 & 0.19 & 0.158 & 0.216 \\
\hline rs9818870 & 0.03 & 0.000 & 0.060 & 0.17 & 0.140 & 0.199 & 0.16 & 0.129 & 0.183 \\
\hline rs9982601 & 0.08 & 0.015 & 0.135 & 0.14 & 0.112 & 0.165 & 0.13 & 0.108 & 0.157 \\
\hline
\end{tabular}

Table 4: Risk allele SNP frequencies in Maori and non-Maori. Reported SNP frequency data is from the Malmo Diet and Cancer Study ${ }^{90} . C I=95 \%$ Confidence Interval, SNP = Single Nucleotide Polymorphism. Red highlighting indicates SNPs with significant differences in frequencies. 


\subsection{GRS Distribution}

Total Population GRS Distribution

The variation in GRS for the total population $(n=420)$ followed a normal distribution, with a mean of 1.45 and standard deviation of 0.180 .

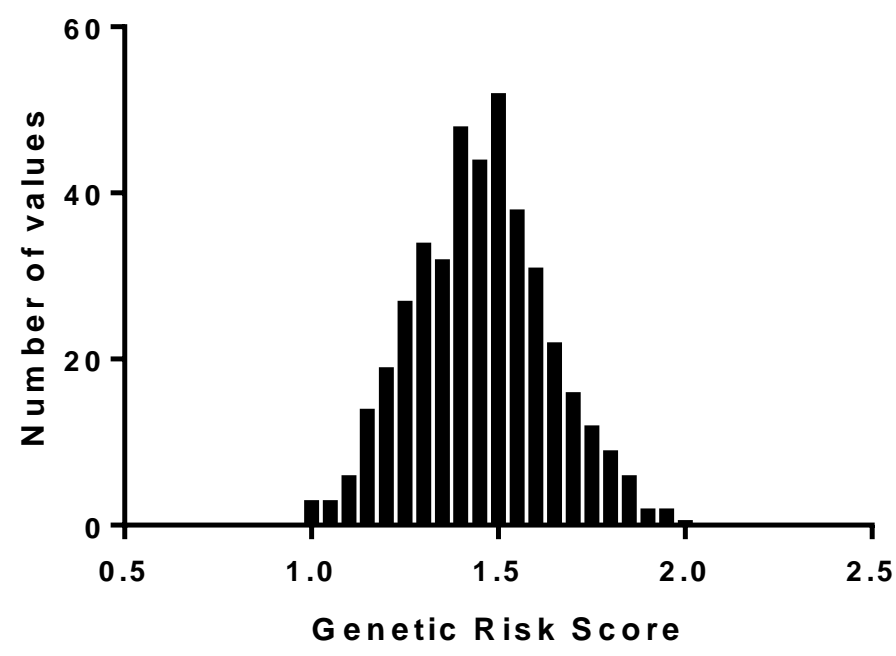

Figure 7: Distribution of GRS values within the total study population $(n=420)$

\subsection{GRS and Age Group}

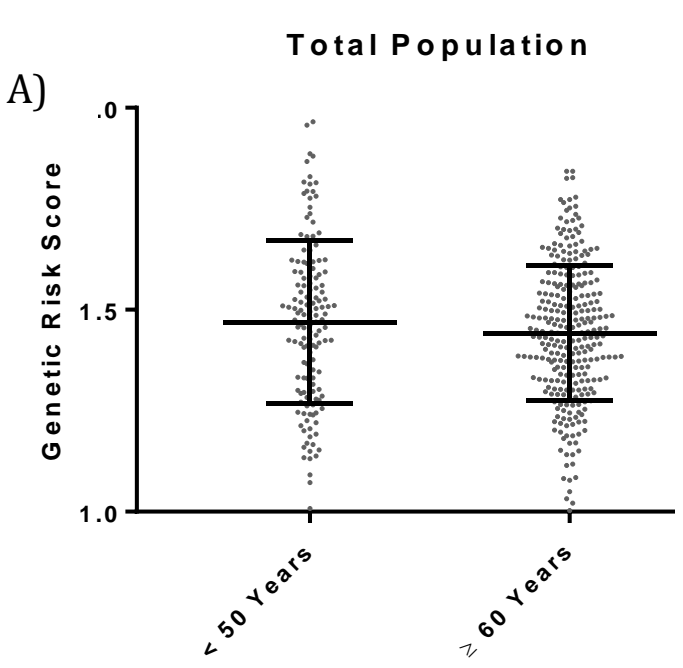

B)

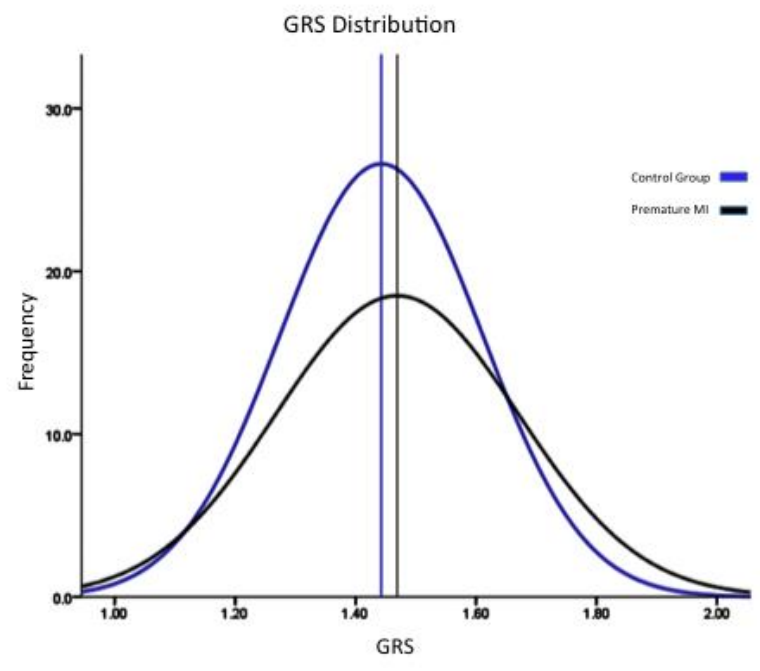

Figure 8: A) GRS vs age group. Mean GRS \pm SD, analyzed with Student's t-test. Premature MI $G R S=1.47$, control group GRS $=1.44, p=0.156 . B)$ GRS distribution comparison in the premature MI cohort and control group.

There was no significant difference in mean GRS between the premature MI and the 60 and older control groups. The premature MI cohort mean GRS was 1.47 compared to that of 1.44 for the control group $(p=0.156)$. GRS distribution was similar in both cohorts. 


\subsection{GRS and Family History}

A)

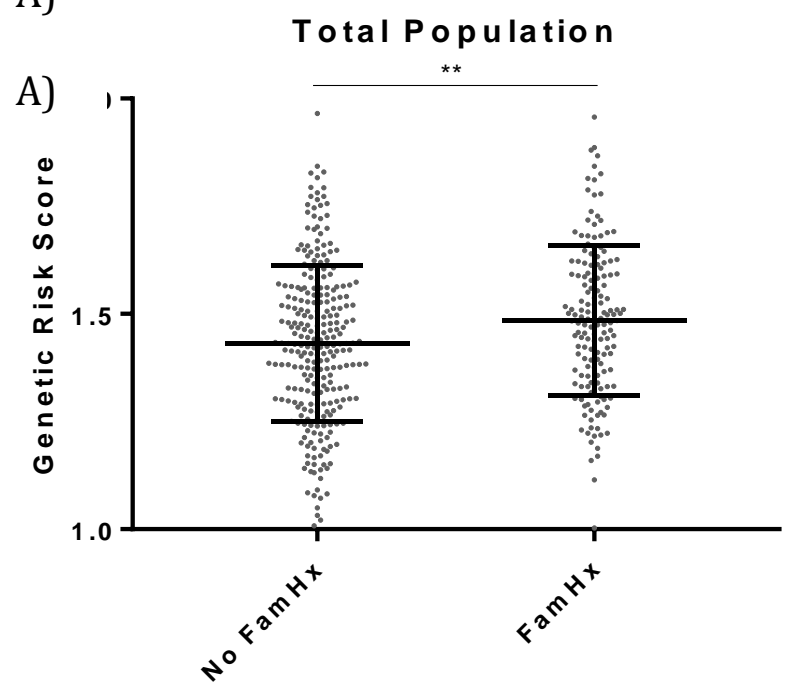

B)

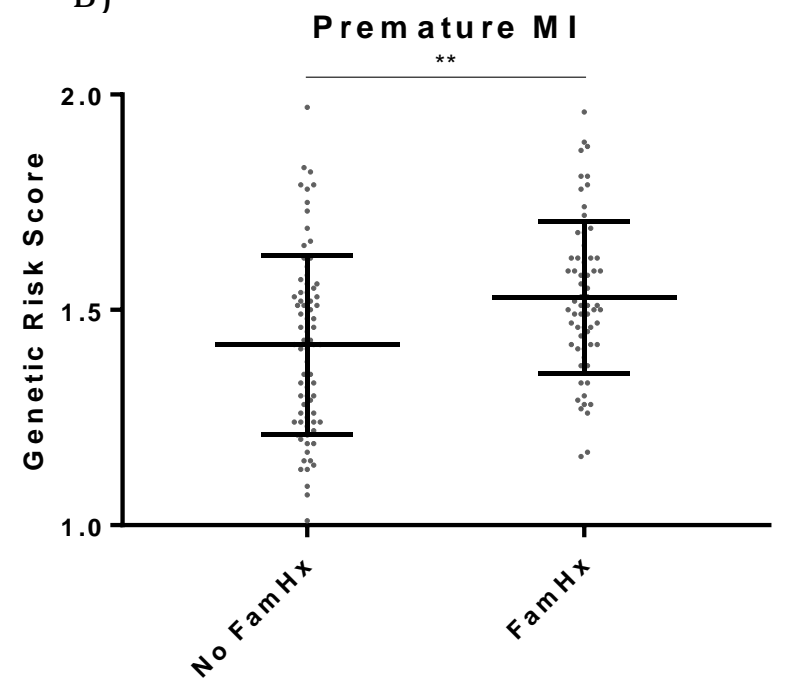

Figure 9: GRS and family history in the total and premature MI study populations. Graphs show mean GRS $\pm S D$. A) The total population, no family history group had a mean GRS of 1.43, with family history GRS = 1.49, $p=0.003$. B) Premature MI cohort no family history GRS $=1.42$, with family history $=1.53, p=0.001$. FamHx = Family History.

Mean GRS was higher in patients with a recorded family history of ACS events versus those with none. This difference was largest in the premature MI group (1.42 vs. 1.53, p = 0.001) and

Patients with Family History of ACS

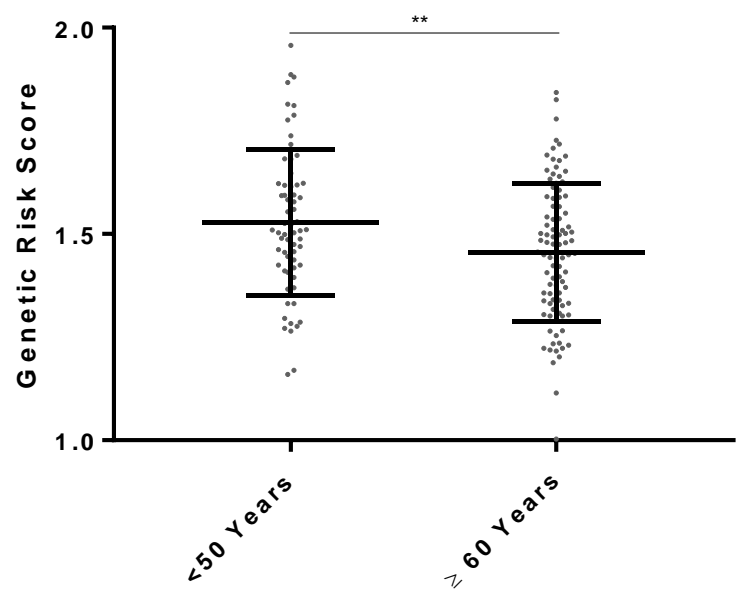
strong in the total population (1.43 vs, 1.49, $\mathrm{p}=0.003)$, however was not significantly different in the over 60 years control group (1.44 vs. 1.46, $\mathrm{p}=0.377)$. Patients with a family history of ACS in the premature MI cohort had a significantly higher GRS than their older counterparts with a family history in the control group (1.53 vs. 1.46, p $=0.008$ ).

Figure 10: GRS between age groups in patients with a family history of ACS. Graph shows mean GRS $\pm S D$. GRS is higher in patients under 50, 1.53 vs. 1.46, $p=0.008$. 


\subsection{GRS and Ethnicity}
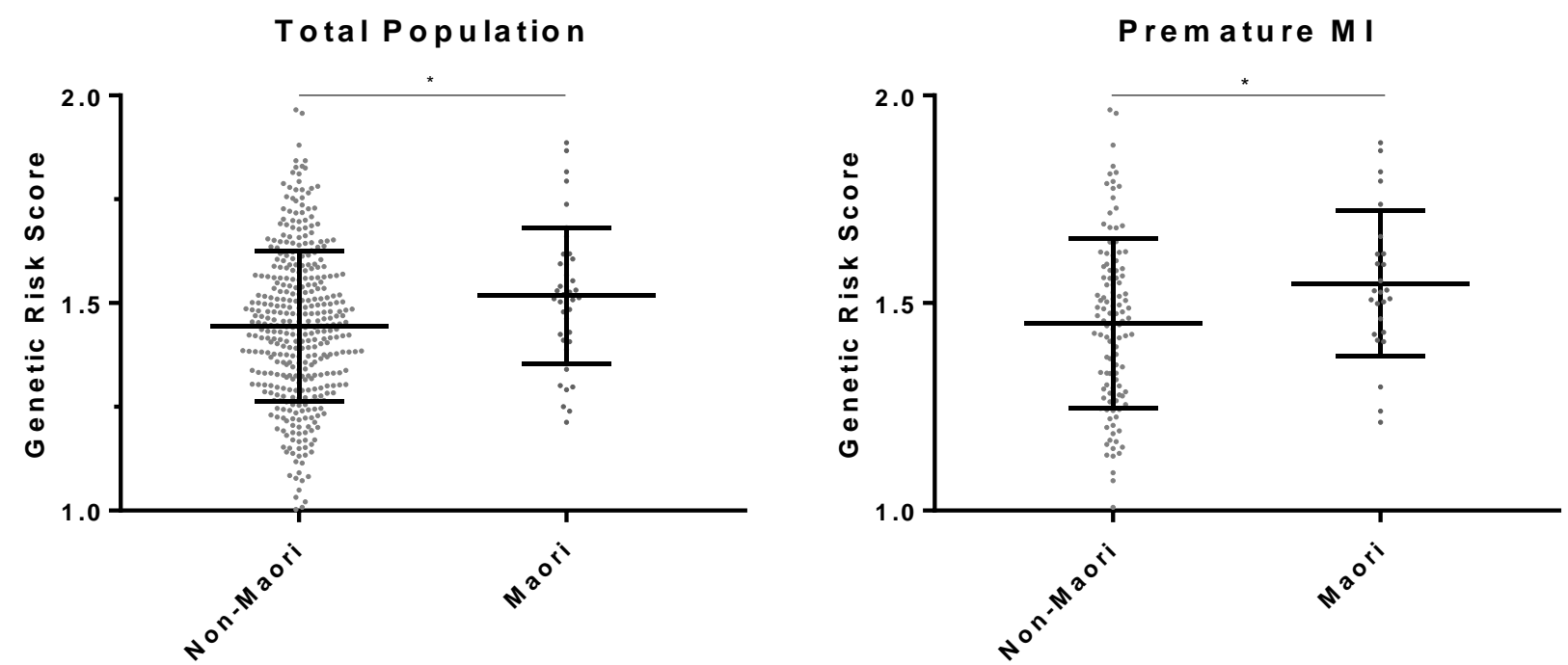

Figure 11: GRS and ethnicity. Maori vs. non-Maori. Graphs show mean GRS \pm SD. A) Total population, GRS is higher in Maori, 1.52 vs. 1.44, $p=0.013$. B) Premature MI cohort, GRS is higher in Maori, 1.55 vs. $1.45, p=0.027$.

In the total population we observed significantly higher GRS in patients of Māori ethnicity when compared to non-Māori (1.52 vs. 1.44 respectively, $\mathrm{p}=0.013$ ). This result was also seen in our premature MI cohort (1.55 vs. 1.45, $\mathrm{p}=0.027$ ). The number of Māori in these groups were low (26 in the premature MI cohort and 40 in the total population), yet even with a small sample size we were able to see a significant difference between the ethnicities. 


\subsection{GRS and Risk Factors}

\subsubsection{Hypertension}

We found no significant difference in mean GRS value between hypertensive and nonhypertensive patients in our premature MI cohort or total population $(p=0.962$ and 0.057 respectively). However, the p-value of 0.057 in the total population is close enough to the alpha value to suggest some association may be present. There was a statistically significant difference in the mean GRS between hypertensive and non-hypertensive patients in our 60 years and over control population. Patients without hypertension had a GRS of 1.47 and those with hypertension saw a value $1.43(\mathrm{p}=0.040)$.
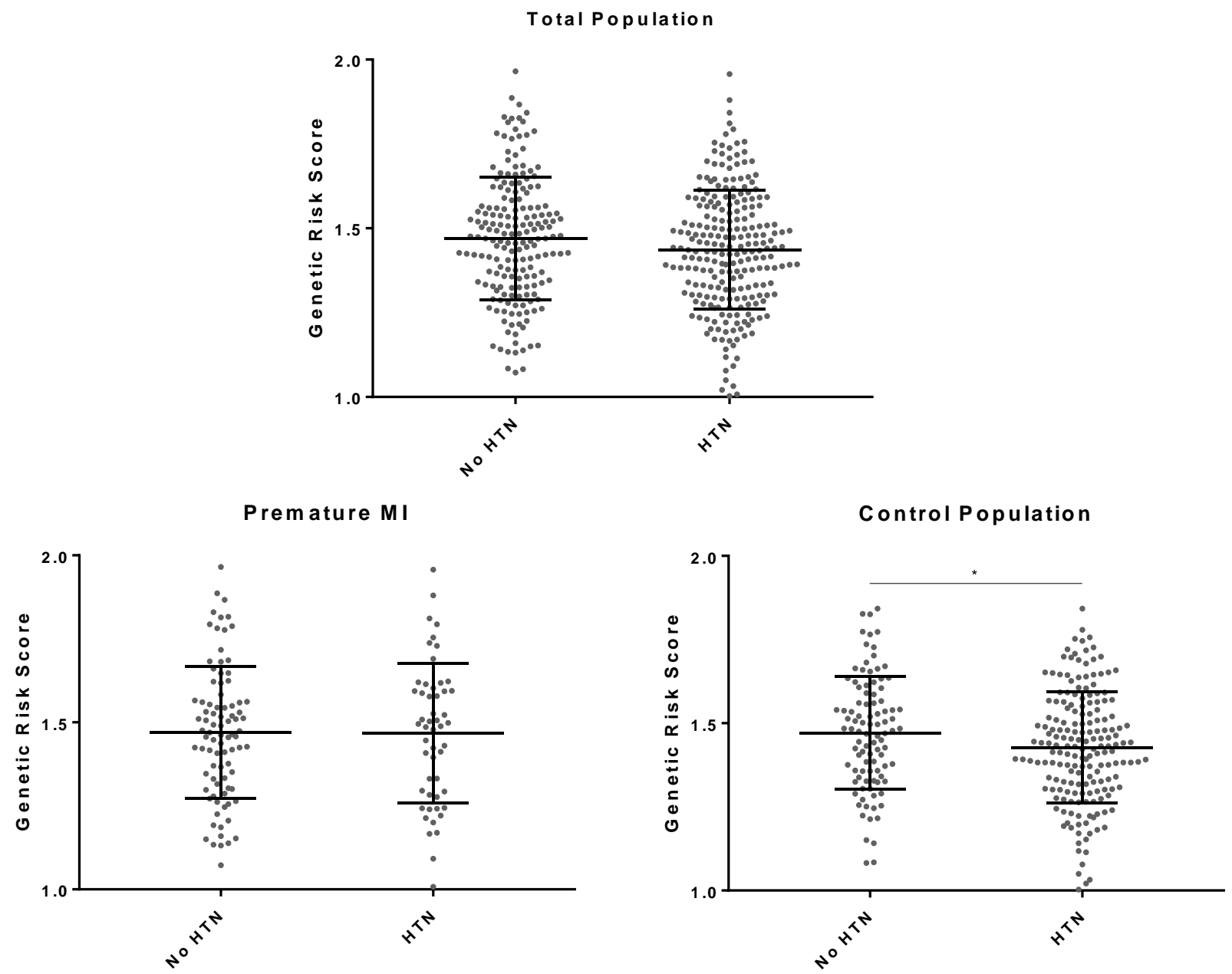

Figure 12: GRS and hypertension. Graphs show mean GRS \pm SD. HTN = hypertension. A) Total population, no significant difference in mean GRS between no HTN and HTN, 1.47 vs. 1.44, $p=0.057$. B) Premature MI, no significant difference, 1.47 vs. 1.47, $p=0.962$. C) Control population, higher GRS in patients with no HTN, 1.47 vs. 1.43, $p=0.040$. 


\subsubsection{Dyslipidemia}

We found no significant difference in GRS between those with and without dyslipidemia, in either of the age groups, nor the total population. In the total population, those with dyslipidemia had a mean GRS of 1.44 as opposed to those without dyslipidemia with a mean GRS of 1.47 ( $p=0.077)$. This was much the same in the premature MI cohort, with mean GRS values of 1.44 and 1.50 for patients with and without dyslipidemia respectively $(p=0.092)$. Once more, these values are close enough to the alpha value to suggest some association may be present and warrant further investigation. In the control population, the mean GRS values were 1.44 and $1.45,(p=0.505)$.

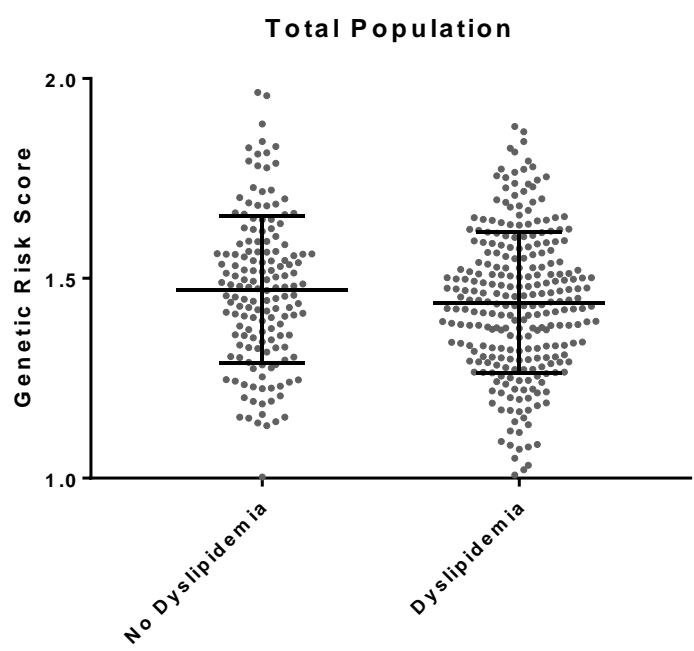

Premature MI

Control Population
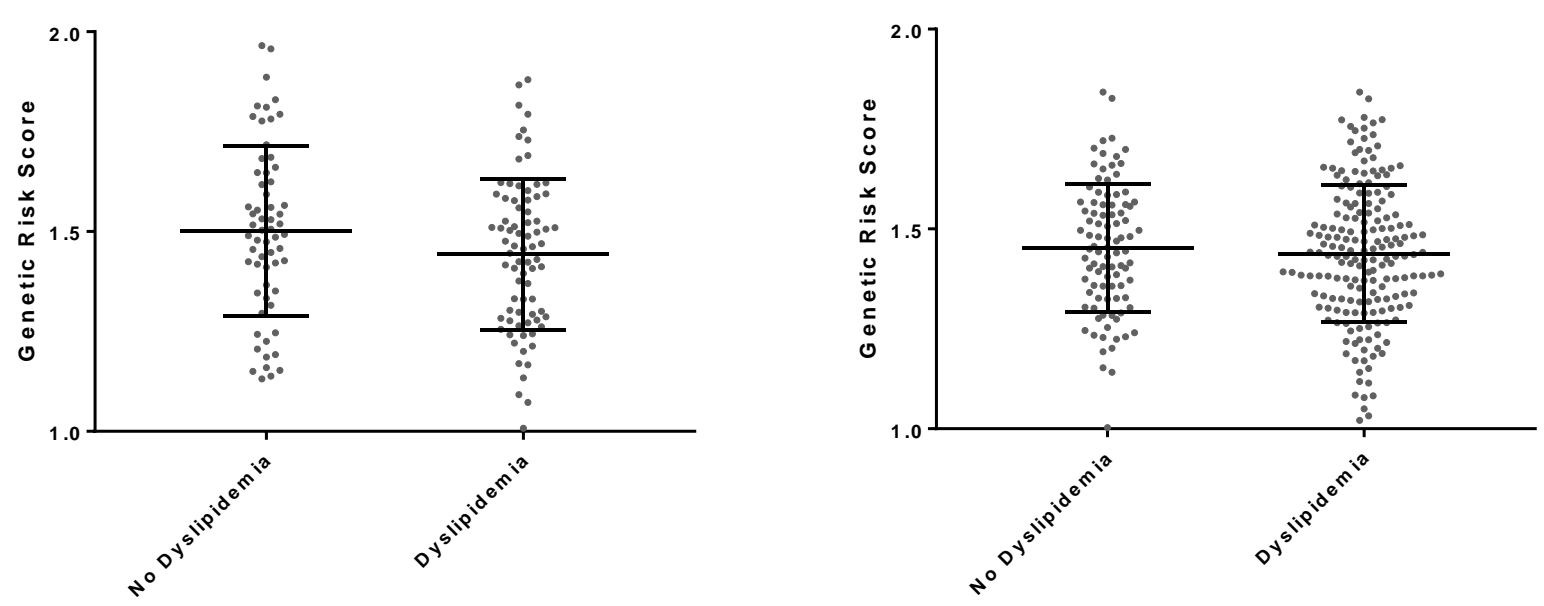

Figure 13: GRS and dyslipidemia. Graphs show mean GRS \pm SD. No significant difference in GRS in all populations between patients with or without dyslipidemia. A) Total population, 1.44 vs. 1.47, $p=0.077$. B) Premature MI, 1.44 vs. 1.50, $p=0.092$. C) Control population, 1.44 vs. 1.45, $p=$ 0.5046 . 


\subsubsection{Diabetes}

In the total population mean GRS values were 1.42 in patients with diabetes versus 1.46 in those without, $(\mathrm{p}=0.117)$. Results were similar in the premature MI cohort, with mean GRS values of 1.43 vs. 1.48 , $(\mathrm{p}=0.336)$. In the control population, mean GRS was 1.42 vs. 1.45, $(\mathrm{p}=$ 0.258). Therefore, using this GRS, diabetes was not seen to be significantly associated with increased genetic risk in our study population.
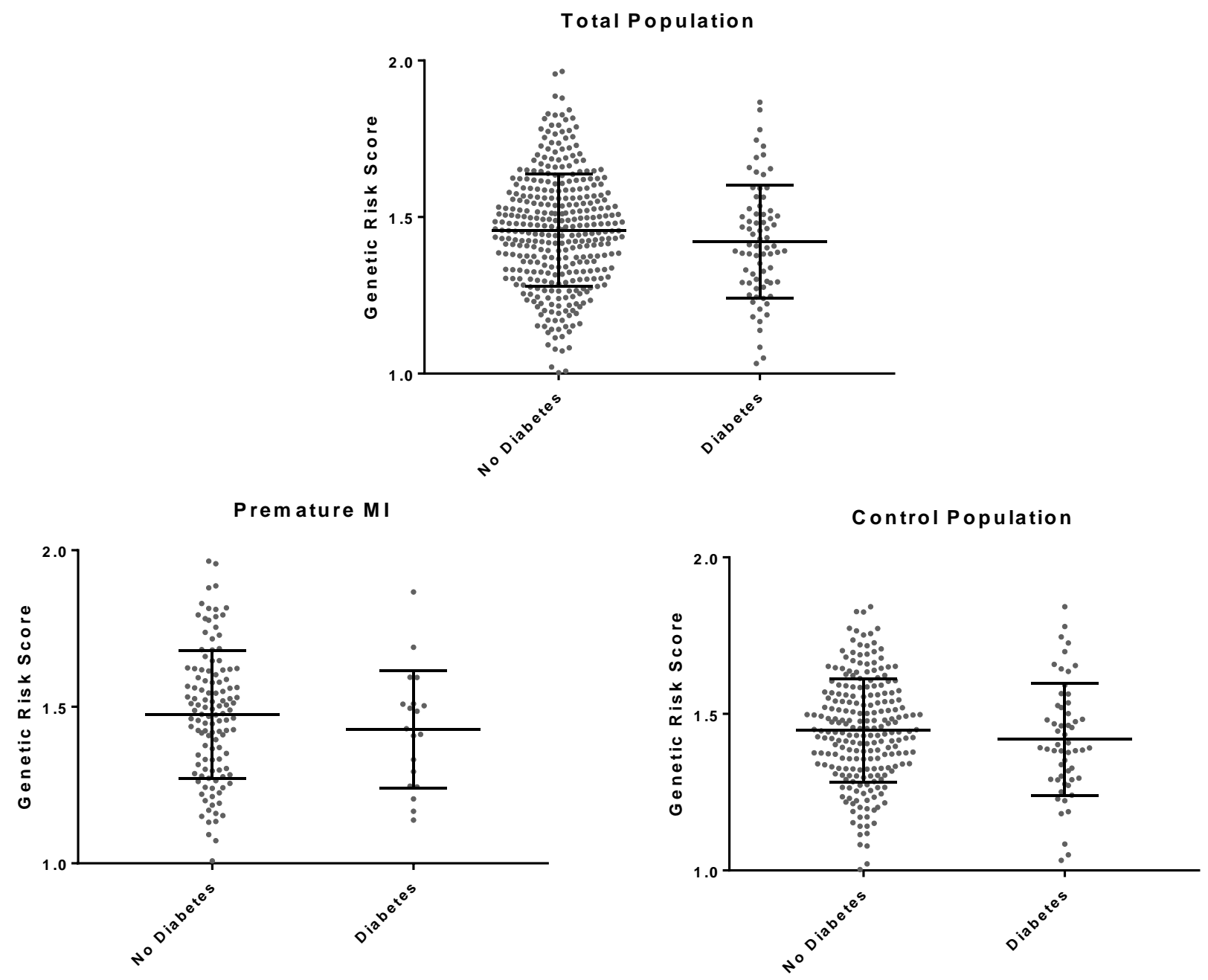

Figure 14: GRS and diabetes. Graphs show mean GRS \pm SD. No significant difference in GRS between patients with or without diabetes in all study populations. A) Total population, 1.42 vs. 1.46, $p=0.117$. B) Premature MI, 1.43 vs. 1.48, $p=0.336$. C) Control population, 1.42 vs. 1.45, $p=0.258$. 


\subsubsection{Obesity}

There was no difference in GRS between those who were obese (BMI $\geq 30$ ) and those who were not. The total population had mean GRS values of 1.45 vs. 1.45 ( $p=0.693$ ). The premature MI cohort had a mean GRS of 1.46 in non-obese patients and 1.47 in obese patients, $(p=0.746)$. The control population had GRS values of 1.43 vs. 1.45 ( $p=0.199)$.

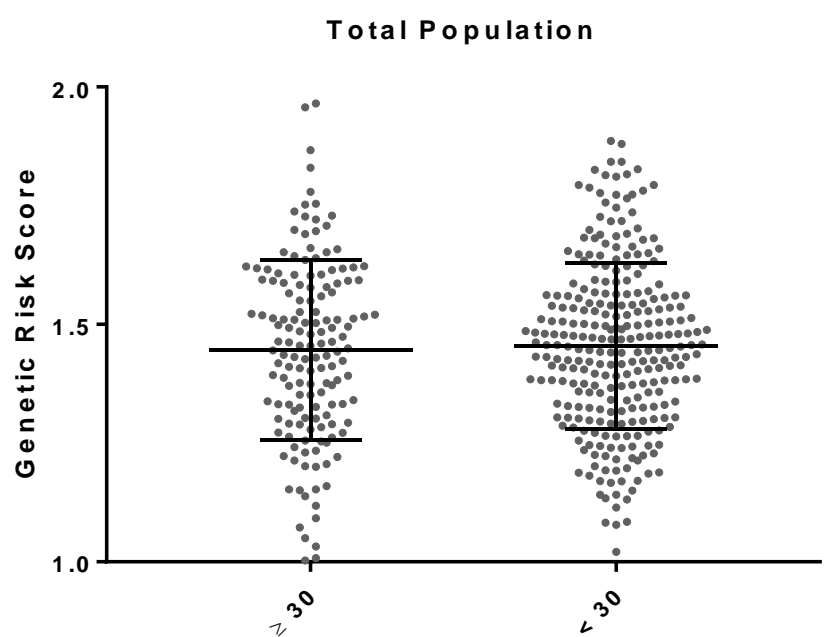

Prem ature MI

Control Population
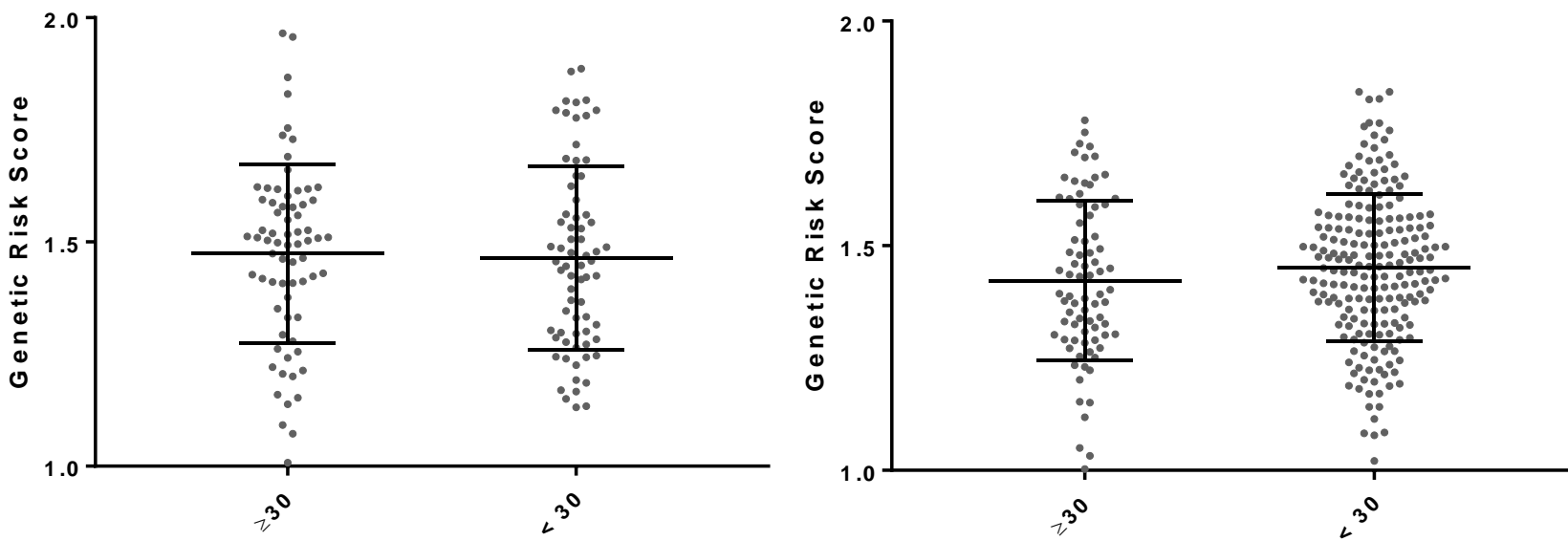

Figure 15: GRS and obesity. Graphs show mean GRS $\pm S D$ vS. Obesity (BMI $\geq 30)$. No significant difference in GRS between obese and non-obese patients, in all of the study populations. A) Total population, 1.45 vs. 1.45, $p=0.693$. B) Premature MI, 1.46 vs. 1.47, $p=0.746$. C) Control population, 1.43 vs. 1.45, $p=0.199$. 


\subsubsection{Combined Risk Factors}

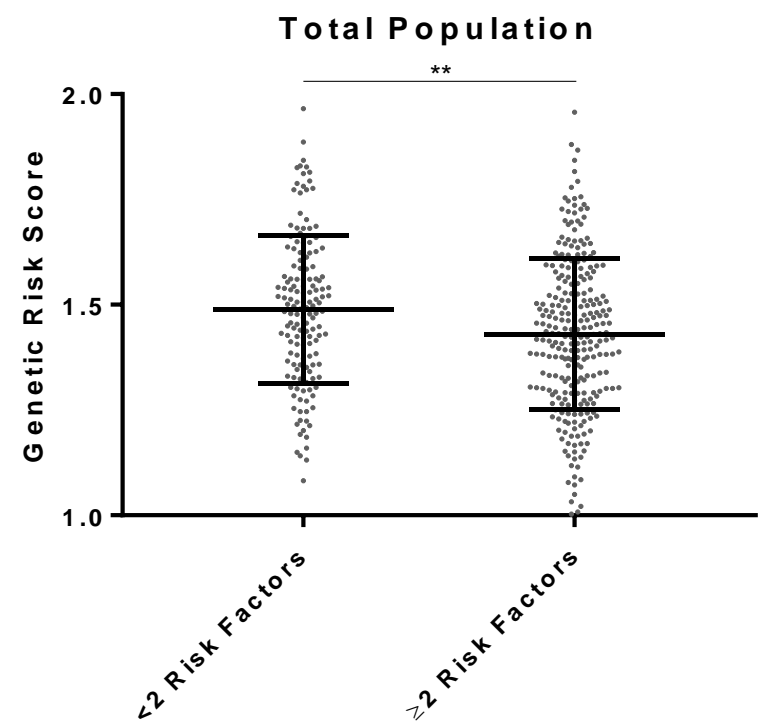

In the total population, patients with fewer (one or less) traditional CAD risk factors (dyslipidemia, smoking, diabetes, obesity and hypertension), were seen to have significantly higher GRS (1.49 vs. $1.43, \mathrm{p}=$ 0.001 ) than those patients with two or more traditional risk factors.

Figure 16: GRS and traditional risk factors. Graph shows mean GRS $\pm S D$. Patients with less than 2 risk factors have an increased mean GRS, 1.49 vs. $1.43, p=0.001$.

Mean Risk Factors per GRS Quintile

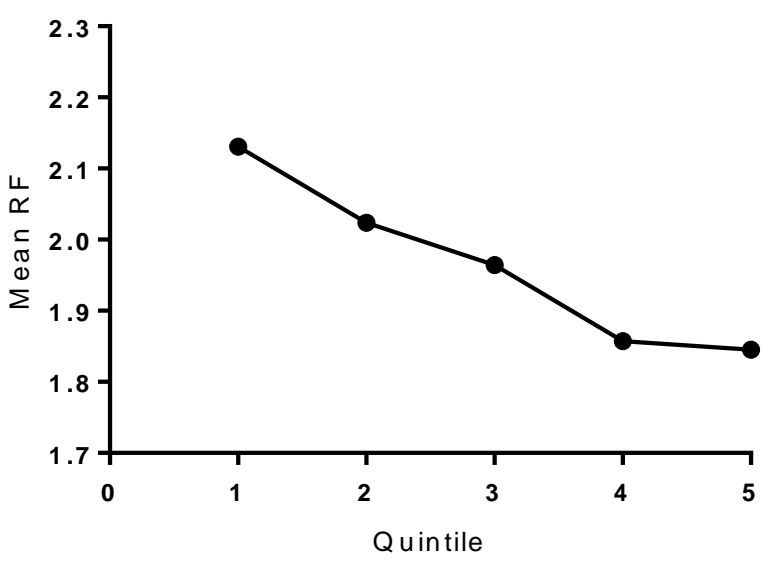

\begin{tabular}{|lll|}
\hline Quintile & GRS Mean & Average RF \\
1 & 1.20207795 & 2.130952381 \\
2 & 1.351901744 & 2.023809524 \\
3 & 1.452087755 & 1.964285714 \\
4 & 1.542067436 & 1.857142857 \\
5 & 1.708266389 & 1.845238095 \\
\hline
\end{tabular}

Figure 17: Mean number of risk factors per GRS quintile. Quintile GRS mean values are in table. Patients per quintile $=84$.

When the patients are split into quintiles according to their GRS values, we can see that there is a trend for lower average numbers of traditional risk factors as genetic risk increases, with a statistically significant difference in average number of risk factors between quintiles 1 and 5 (p $=<0.001)$. 


\subsection{GRS and Gender}

GRS values between male and female patients within our study detected no significant differences in any of the population groups. Total population GRS was 1.43 for females and 1.46 for males, $(p=0.079)$. This value is close enough to the alpha value to suggest that an association may be present, however the results from the two sub groups do not strictly support this. The premature MI cohort had mean GRS values of 1.44 for females and 1.48 in males $(\mathrm{p}=0.425)$. In the control population, mean GRS values were 1.42 versus $1.45(\mathrm{p}=$ $0.164)$.

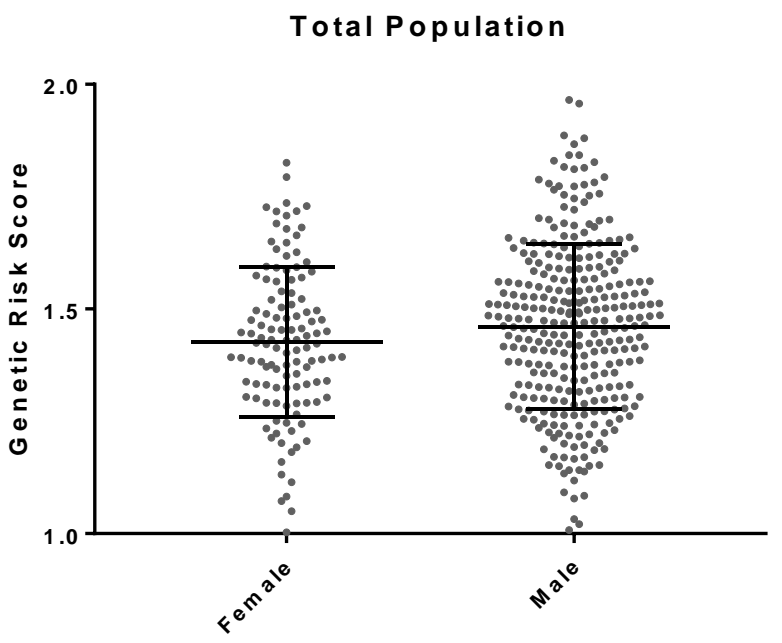

Premature $M I$

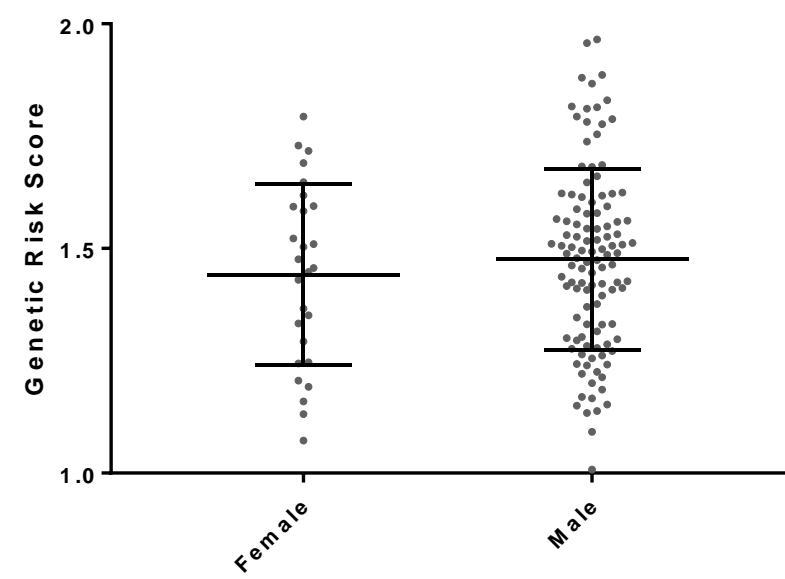

Control Population

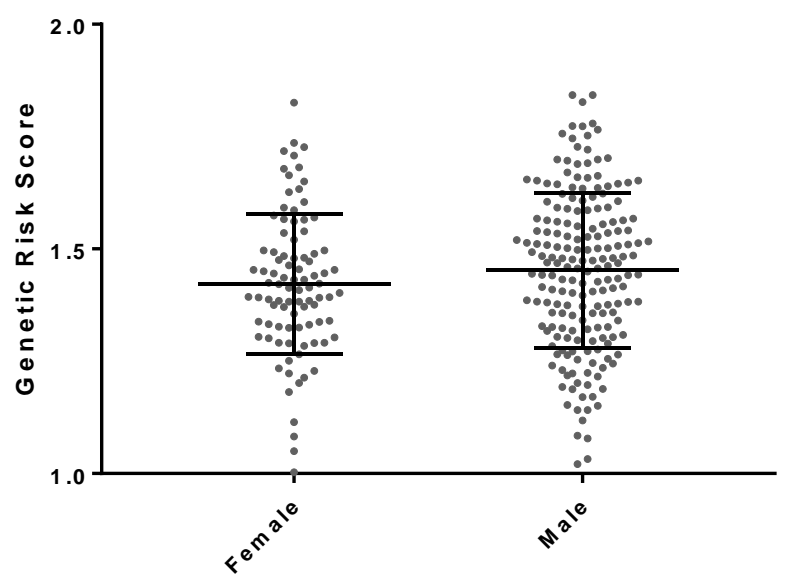

Figure 18: GRS and gender. Graphs show mean GRS \pm SD. No significant difference in GRS between females and males, across all of the study populations. A) Total population, 1.43 vs. 1.46, $p=0.079$. B) Premature MI, 1.44 vs. 1.48, $p=0.425$. C) Control population, 1.42 vs. 1.45, $p=0.164$. 
Hadley Northcott 


\section{Chapter 4 - Discussion}

There were a number of significant differences between the patients within our premature MI cohort and those over 60. The premature group had a higher proportion of male subjects, patients with Māori ethnicity, patients with a family history of CAD, obesity, and current smokers than the over 60 years old patients. The patients in the older group were more likely to have a history of hypertension and dyslipidaemia. We found that the mean GRS was not elevated in the premature MI cohort versus the over 60 years MI patients. Mean GRS was slightly higher in patients with a family history of coronary disease, in Māori patients and in patients with fewer than 2 traditional risk features. GRS was not associated with individual traditional risk factors, including dyslipidaemia, hypertension, diabetes, obesity or gender. The frequency of the individual SNPs in our total cohort were similar to those seen in overseas populations, however a small number of differences were observed. Limited differences were seen in SNP frequencies between the two age groups, however we did see significant differences in SNP frequencies between our Māori and non-Māori populations.

We did not observe a significant difference in mean GRS between the premature MI cohort and our 60 and over control group, with the premature MI cohort having a mean GRS of 1.47, compared to 1.44 in the control group. Thus the GRS we have used does not appear to be significantly detecting factors that contribute to earlier onset MI. The SNPs used in the construction of the GRS will be playing a role in this. The SNPs included within this panel have come from all age population-based GWAS studies 66,70,90,148 and validated in clinical trials of statin therapy ${ }^{89}$. This approach biases towards common factors associated with CAD, most of which occurs later in life, rather than the processes more explicitly involved in premature MI. As yet, limited GWAS have been run exclusively on patients who experience premature MI. This creates a limitation in the SNP panel that we use, as in lieu of traditional risk factors, the onset of ACS at a young age may differ in terms of rates of progression and processes involved, which raises the question if in fact there are different mechanisms behind the exacerbated advancement of the disease. Hence, different SNPs could be implicated in premature MI than those that we have found from studies involving patient cohorts in which a majority are older in age. 
A number of other studies have looked at the interaction between genetic risk and premature onset of CAD, however still using SNPs discovered in GWAS encompassing very broad patient demographics. Among these, Tada et al, using a similar GRS calculation in 23,595 patients, found that GRS had utility as an addition to existing ACS prediction. They saw that GRS was independent of family history and was useful in determining risk in younger patients ${ }^{83}$, however their criteria for young ACS differed from ours, using subjects with an average age substantially higher than our premature MI cohort, and their patient population was much larger, as such it is hard to draw direct correlations between the two studies. Vecoli et al and Labos et al, using panels comprised of SNPs varying to those used within our study, saw significant correlations with premature onset 79,87 . These results were backed up by Hindieh et al who found that individuals with higher GRS were at greater risk of having multi-vessel CAD, as assessed by coronary angiography in 763 premature ACS patients. ${ }^{76}$. The SNP panel used by Vecoli et al differed greatly in comparison to the panel used within our study, using 44 SNPs, each different from those contained within our panel, yet with a number in similar gene families and pathways. Labos et al used a SNP panel with many similarities to the one within our study, with 26 of their 30 SNPs matching. However, they used an alternative method of calculating their score, using unweighted sums.

The variation in methodology in regards to calculating the genetic risk is apparent between different studies, with the main divide appearing to be between the uses of scores weighted by odds ratios versus those that use a simple count method. Previous studies have addressed this issue, and their results showed that a GRS that uses a weighted method outperforms a GRS that uses a simple count method ${ }^{149,150}$. One issue with this however, is that these weightings are derived from odds ratios that were calculated from studies involving overseas populations. As a result of this, we are unsure how applicable these odds ratios are to the New Zealand population, which, as has been stated previously, is comprised of a unique grouping of ethnicities. To answer this question accurately, a much larger cohort based study would be required within the NZ setting, and this was outside the scope of this thesis. Our SNP frequencies, whilst showing some differences, are in similar ranges to that which is seen in these overseas based studies. While this doesn't exactly speak of the similarities in odds ratios between 
the populations, it shows the groups are not drastically different as far as we can see, and therefore we feel the weightings are applicable within our study.

Hence, we can see that there is still much variation between all of the study designs being used in the search to find a robust GRS method. The lack of a statistically significant correlation between GRS and premature MI within our cohort may reflect a genuine similarity in genetic risk between these two study groups, or it could possibly be due to panel design or study size. Also, both of these age groups in our study had a large percentage of patients who had a number of traditional risk factors. This may well have resulted in the effects of increased genetic risk being masked by the effects of major risk factors such as smoking and obesity.

Modifiable cardiac risk factors were more common within our premature MI cohort. The patients under 50 years old were much more likely to be obese and to be current smokers. Of the premature MI population, 62\% of the patients had more than one traditional risk factor and only $10 \%$ had no traditional CAD risk factors. Thus, although one aim of this study intended to assess the level of genetic risk in premature MI patients with low traditional risk for CAD, the high levels of risk factors made finding the true impact of genetics on premature ACS difficult. From the analyses of the risk factors within the age groups however, it appears smoking and increased BMI are likely significant drivers of premature ACS events, as well as Māori ethnicity and a family history of CAD.

In New Zealand, due to the substantial adverse effects that tobacco smoking has on the health system, a large initiative for the country to be "Smokefree by 2025" is well under way. The NZ population has seen steady rates of smoking cessation in all demographics, apart from Māori woman 151. Unfortunately, the highest rates of smoking are seen in populations that also experience high rates of ACS events, and within our study population appear to be one of the groups that are most genetically at risk of MI, this group being Māori under 50 years of age (as discussed below). Smoking rates in Māori are significantly higher than rates in non-Māori 5,151, and the percentage of smokers under 50 years of age in our cohort was much higher than in the patients 60 years or 
more. Hence, smoking is still a large driver that appears to be contributing significantly to ACS rates, especially within our premature MI cohort and within Māori.

It is likely that to truly assess the effects of genetic risk for CAD within these populations, we would need to recruit a cohort of patients that do not smoke, have a healthy BMI and do not possess any other cardiac risk factors. There is also the possibility that adjustments may be required to the GRS itself. A specific GRS looking for early onset MI may require a different set of SNPs, potentially from a more targeted population of patients who suffer severe early onset ACS events and have a strong family history of the disease, and do not possess traditional risk factors.

Many of the SNPs were present in similar frequencies between the total study population and the reported frequencies obtained from the Malmo Diet and Cancer Study (MDCS). However, there were four SNPs that were present at significantly different frequencies between the two populations. These were rs10455872, rs46522, rs9349379 and rs964184, which are associated with the LPA, UBE2Z, PHACTR1 and APOA5 genes respectively. The $L P A$ gene, coding for Lipoprotein (a) [Lp(a)], is a wellknown contributor to atherosclerotic processes. Lp(a) levels in blood plasma are mainly regulated by the $L P A$ gene, with dietary and environmental factors not greatly affecting blood concentration. $\mathrm{Lp}(\mathrm{a})$ is involved in thrombogenesis and acts in inflammatory cell recruitment to the vessel walls, which can lead to smooth muscle cell proliferation 111113. The UBE2Z gene codes for an ubiquitin conjugating enzyme which is involved in protein ubiquination during proteolysis 152,153. The PHACTR1 gene encodes the Phosphatase and Actin Regulator 1 protein. PHACTR1 has been seen to be involved in atherosclerosis, as it is regulated in macrophages and endothelial cells by atherogenic stimuli. The rs9349379 risk allele has effects on PHACTR1 expression in macrophages that are similar to inflammatory stimuli ${ }^{106}$. The APOA5 gene encodes apolipoprotein A$\mathrm{V}$, a protein that influences plasma triglyceride levels ${ }^{154}$. The genes that these SNPs are associated are all involved in processes that have been linked to CAD.

We would expect some variation in SNP frequency between these study groups, as the MDCS was undertaken in a healthy prospective cohort 90 whereas our study involved patients with already proven CAD. Hence, we expected that our population, with pre- 
existing disease, would have higher frequencies of the risk alleles within the GRS panel. This, to some extent was observed, however, given a larger sample size, it is likely that more significant differences may be visible between the two cohorts.

SNP frequencies between the premature MI cohort and the 60 years and over control group were very similar. Realistically, we do not have a sufficiently sized study population to make any confident conclusions on the SNP frequencies between these age groups, as the study is not specifically powered for this. This study was designed to assess the varying genetic risk between premature and older MI using a multi-locus GRS, hence looking at differences between individual SNPs is slightly outside the scope of the original design. This small sample size results in wider confidence intervals for the SNP frequencies, especially within the premature MI cohort. A larger population would give smaller confidence intervals, enabling more confident extrapolation of results. However, the results from our study can serve as beneficial pilot data, in noticing general trends and SNPs which have a large difference in risk allele frequency.

However, one SNP was significantly different between the two age groups, this being rs3184504. The risk allele at this loci was less frequent in the premature MI population. The rs3184504 SNP is associated with the SH2B3 gene. This gene codes for an adapter protein that regulates a number of cell signaling pathways, including some involved in inflammation, cell migration and hematopoiesis ${ }^{131,155}$. Given that the premature MI population is regarded as our more "at risk" sub group in regards to genetic risk, the fact that the risk allele for this SNP is present at lower frequencies than the control population raises a number of questions. While this result may truly show a difference in the risk allele frequency between premature and older MI populations, this will need to be validated in a larger study population. Thus, we cannot confidently say that any individual SNP is having a drastic effect on the occurrence of premature ACS events. This shows the importance of having a multi-locus GRS, as fluctuations and noise at individual loci can be ironed out, and the combined effects of the high risk alleles within the SNP panel can be seen and analysed more confidently.

Despite not predicting early onset MI, we found that GRS was higher in those with family history, especially within the premature MI cohort. This is a slight paradox, as 
family history is linked to early onset MI55. Therefore, this suggests that the GRS is detecting some aspects of genetically inherited risk within our population. This association is consistent with the understanding that ACS events amongst relatives have a genetic component and are not always due to environmental factors and pattern behaviour between family members. The link between GRS and family history has been seen in previous studies. Hindieh et al reported a significant correlation between family history and GRS in angiographically assessed patients ${ }^{76}$, and Tada et al saw similar results within their Malmo Diet and Cancer Study data ${ }^{83}$.

Patients in the control group did not show a significant correlation between family history and genetic risk, suggesting that hereditary factors may have their largest effect in younger individuals. To support this finding, we saw that patients with a family history of CAD in the premature MI cohort had a significantly higher GRS than their older counterparts with a family history in the control group, indicating once more that the hereditary component appears to be stronger in those patients that are afflicted by ACS at a younger age. One argument to explain this is that family history is present in two variations. One being the hereditary aspect that our study is interested in, which appears to be present in higher abundance within the premature MI cohort, and the other being due to environmental factors within families, which shows limited correlation with genetic risk, and appears to be more apparent in MI cases that occur after 60 years of age. Family history was defined as any direct blood relative who had angina, MI or sudden cardiac death before the age of 55, as described by the American College of Cardiology 156 . Previous studies have highlighted the heritability of CAD, in parents and their children ${ }^{55}$ as well as in siblings and twin studies ${ }^{54,58}$. With this data we can show a genetic link between this association, and now have the ability to use the information encoded by the SNPs in the panel to further study what genes and processes may be involved.

Māori MI rates are consistently high, at a level disproportionate to the rest of the NZ population, with Māori ischaemic heart disease mortality rates at 106.2 per 100,000 population, compared to that of non-Māori at 58.04. While researchers have often hypothesized that there may be a genetic link to the increased rate of MI in Māori, a common argument has been that environmental and socioeconomic factors play more 
of a role ${ }^{7}$. These include factors at the individual level, such as diet and exercise, as well as population level inequalities with access to the healthcare system ${ }^{8}$. There is a complex interplay of factors occurring, however we observe what could be, upon careful exploration, a more tangible link between genetics and the increased rate of MI in our Māori population. The mean GRS in the Māori population within our study did have a statistically significant increase over the non-Māori population. Highlighting that with this panel, we have the ability to elucidate increased genetic risk in Māori. In conjunction with existing cardiovascular risk scoring, the GRS technique could add vital information in identifying individuals at risk of ACS events.

There were a number of SNPs within our Māori population that were present at different frequencies to what was seen in non-Māori. This clarifies the source upon which the difference in the mean GRS results from. Interestingly, while there are a number of risk alleles that are more frequent in Māori, which fits in with our initial hypothesis of increased genetic risk in Māori and our other results, there are also a few SNPs that see a reduced frequency of the risk allele in Māori. Overall, the SNP frequencies within our study show substantial variance across the entire panel between Māori and non-Māori. This variance is not entirely captured by the difference in the means of the GRS, as the reduction in frequency of some risk alleles will be softening the effect of the increase in the risk alleles for a number of the other SNPs.

Six SNPs were present in significantly different frequencies between the Māori and nonMāori populations. These changes in frequency include both more abundant and less abundant rates of certain SNPs in the Māori population. Although more variation appears to be present when looking at the graph, using $95 \%$ confidence intervals, we can only see significance in six of these. This is most likely due to the size of the study population. As discussed earlier, a larger cohort would likely reduce the size of the confidence intervals, allowing us to be more confident in differences observed between the study populations. The six SNPs that were significantly different are; rs216172, rs2259816, rs3184504, rs3798220, rs635634 and rs9818870 which are associated with the SMG6, HNF1A, SH2B3, LPA, ABO and MRAS genes respectively. 
SMG6 codes for the Telomerase-binding protein EST1A protein. This protein functions in both RNA surveillance and telomere maintenance ${ }^{140 .}$ The rs2259816 SNP is associated with the $H N F 1 A$ gene, a transcription factor believed to be implicated in some forms of diabetes, liver function and interactions with CRP and other acute phase response proteins ${ }^{157}$. The rs3184504 SNP, in which we see a much lower risk allele frequency in the Māori population is associated with the SH2B3 gene. This gene codes for an adapter protein that regulates a number of cell signaling pathways, including some involved in inflammation, cell migration and hematopoiesis 131,155. The LPA gene, coding for Lipoprotein (a) [Lp(a)], is a well-known contributor to atherosclerotic processes. Lp(a) levels in blood plasma are mainly regulated by the LPA gene, with dietary and environmental factors not greatly affecting blood concentration. Lp(a) is involved in thrombogenesis and acts in inflammatory cell recruitment to the vessel walls, which can lead to smooth muscle cell proliferation ${ }^{111-113}$. $A B O$ codes for the histoblood group $\mathrm{ABO}$ system transferase protein. This protein determines what the $\mathrm{ABO}$ blood group of an individual will be 119,120 . ABO blood group may possibly be linked to MI through the relative abundance of clotting factors present between groups. The MRAS gene codes for the Ras-related protein M-Ras protein. This protein is ubiquitously expressed and is involved in signal transduction. It is well implicated in a number of cancers and developmental disorders, however, as yet its function in CAD remains unknown ${ }^{102 .}$

The differences in allele frequency at the rs3798220 (LPA) and rs9818870 (MRAS) loci are most likely due to the very low overall frequency of these SNPs. The risk alleles for rs2259816 (HNF1A) and rs635634 (ABO) were more frequent within the Māori cohort and the risk alleles for rs216172 (SMG6) and rs3184504 (SH2B3) were less frequent within the Māori cohort. A number of these processes have been implicated in the pathophysiology of atherosclerosis, while some processes are not well documented in this area at all. The observation of the inconsistency in the risk allele frequency between these ethnicities opens the door for further research into the incidence and function of these loci. In turn, this could help us with tools for diagnoses and screening strategies specific to our NZ population, as well as possible therapeutic targets. 
The fact that we saw significant differences in six of these SNPs, even though we had a low number of Māori patients and therefore relatively wide confidence intervals, gives promise to the idea that there could be a number of SNPs that are present in statistically significant different frequencies, as alluded to visually on the graph, within a larger Māori population. With the observation of high rates of ACS in Māori 4,5, it can be hypothesized that they could possibly be more genetically at risk than non-Māori. This is suggested by the difference in overall mean GRS between Māori and non-Māori, and then reiterated by the variance in individual SNP frequency.

Hypertension, dyslipidaemia, diabetes and obesity are all well-established risk factors for CAD that contribute to the initiation and progression of atherosclerotic lesions through a number of mechanisms 22,39-42,48-50. We saw no significant association with GRS in the total population, as well as within the two age groups, when looking at dyslipidaemia, diabetes and obesity. Therefore it appears that this GRS does not have the ability to detect genetic risk in relation to the presence or absence of these risk factors. There was a small, yet significant, correlation in hypertensive patients in the 60 years or older group. In this group, we saw that non-hypertensive patients had a higher mean GRS $(p=0.040)$. The premature MI cohort and total population saw no significant difference in regards to hypertension. The $p$-values in relation to total population hypertension $(p=0.057)$, total population dyslipidaemia $(p=0.077)$ and premature MI dyslipidaemia ( $p=0.092)$ were close enough to the alpha value $(0.05)$ to suspect that some form of association might be present. However, with these results not being significantly different, and the real differences in GRS values only being between 0.03 and 0.06 apart, the difference in GRS in these groups would likely not result in any phenotypical differences that would be clinically significant. With that being said, these associations may warrant further investigation in larger study populations.

Overall, we see that individual traditional risk factors do not appear to correlate to a significant level with this GRS. This GRS was developed to look at genetic risk of ACS, not at the individual factors that are known to increase risk. The GRS panel also includes a number of SNPs in pathways outside those of previously established risk factors. Hence, it is not overly surprising that the score does not correlate with the individual risk factors. 
When we group the data into patients with fewer traditional risk factors versus those with substantial risk according to traditional features, we see that there is a relatively strong association between low traditional risk and increased genetic risk. Also, when we look at this question from a slightly different angle, by breaking the total patient population into quintiles based upon GRS values, we can see a correlation between increased genetic risk and decreased number of traditional risk factors.

In the total study population, patients with fewer risk factors had a higher GRS. This suggests the GRS is capturing some elements of risk that are independent of traditional risk features and supports the idea that genetic risk scoring may have additive value to current risk scoring. A number of studies have demonstrated that a GRS has additive effect on top of traditional risk factor screening ${ }^{72,83}$, which alludes to the ability of GRS to identify mechanisms outside those of established risk factors. Our results are consistent with this argument and suggest that the presence of significant traditional risk factors may not be required in order for an individual to develop CAD. One caveat with this result is that the number of premature MI patients with one or fewer risk factors was small (less than 28\%) as the majority of the younger patients had considerable risk from traditional factors, which presumably would be driving their disease. Hence, specifically targeting a larger group of premature MI patients, with minimal risk as determined by traditional risk factors, would be desirable in order to validate this result.

Being able to identify these low traditional risk, high genetic risk patients could prove to be very beneficial. These individuals are often not on any preventative therapies and can often become complacent, believing they have no risk of ischaemic events. Therefore, the successful inclusion of genetic data into routine CVD risk assessment could cast a wider net, identifying more 'at risk' patients, and therefore enabling clinicians to start preventative strategies earlier.

One problem that arises through analysis of the data based on having one or fewer risk factors, is that each risk factor could have widely varying weight on the progression of disease, and this model does not account for which factor is present. This would grow in 
complexity once more when we account for the variance in severity of the indvidual risk factors as well. For example, someone who smokes one ciggarette per day is classified as a 'Smoker' in the same manner as an individual who smokes 10 cigarettes per day, regardless of the large difference in the effects on their health over time. A more comprehensive risk factor scoring system may enable us to correct our data to a larger degree, in order to elucidate more specific genetic drivers of disease.

Within our population we saw that the number of ACS events was not equal between genders. This is shown by $80.7 \%$ of our premature MI cohort being male, along with $71.9 \%$ of the study population as a whole. Women tend to suffer from a primary ACS event approximately 10 years later than men, and also tend to experience worse outcomes ${ }^{53}$. We looked to test if our SNP panel could detect any form of genetic basis behind these gender differences. In regards to this question, we were unable to find any significant difference in the mean GRS ( $\mathrm{p}$-value $=0.079)$, with males having a score of 1.46 and females a score of 1.43 . From this, there are a number of possible conclusions; one being that our SNP panel did not focus on the right markers to answer this question, as the GWAS studies that found these loci did not design their studies with this query in mind. As such, we find no SNPs in our panel within loci that are commonly associated with gender differences, i.e. the allosomes. Another possible conclusion, that has been assessed in previous studies 53 , is that the gender difference is in fact not due to direct genetic influence, and is instead a secondary result of hormonal and physiological differences between males and females.

\section{1 - Limitations}

One limitation in this study, as discussed previously, is the sample size. While it was sufficiently sized for the primary analyses of comparing GRS between age groups and in associations with the whole population, upon breaking down the sample for subanalysis we encountered problems in regard to statistical power. This is because of the patients that were available to be recruited into the study. While it would have been beneficial for our study if more premature MI patients had presented during the 3.5 year recruitment period, this did not prove to be the case. Hence, a larger cohort would 
enable us to further break down these results i.e. look at GRS in patients of different ethnicities and who have low traditional risk.

Another element that was limiting in this study was the number of risk factors present throughout our study population. Subsequently we had difficulty in seeing genetic risk effects. This is possibly due to being masked by the effects of risk factors such as smoking, obesity, dyslipidaemia and diabetes. Hence, further study of genetic risk would ideally be carried out in a patient population with negligible traditional risk.

The patients included in this study had verified CAD, allowing the comparison of young and old ACS patients. This study design enabled us to look at differences between premature and older MI patients and try to observe variances in these different disease states. An interesting comparator study would be to compare these results to patients who have no evidence of CAD, in order to create a baseline of genetic risk.

This GRS is calculated from the presence or absence of the 27 SNPs in the panel. The number of risk alleles present $(0,1$ or 2$)$ is then weighted by the log of the odds ratio for each of the SNPs. A linear sum of these then produces the genetic risk score for each patient. This GRS panel and calculation was adapted from a well-constructed peer reviewed study involving over 48,000 individuals ${ }^{89}$. Different studies use various methods of calculating genetic risk, these differ in both the SNPs that are included and in the way that the score is calculated 71,72,74,78,79,82-84,87,89,158. Hence, different methods could also yield different results. A number of these SNPs are of unknown function, and associations with new SNPs are still being found. Therefore, further optimization of the construction of a genetic risk score is possible, both by further verifying the SNPs used and possibly expanding the panel, and/or by testing different methods of calculating the score and validating in a separate patient cohort. 


\section{2 - Future Directions}

There are a number of possible directions that could be followed from this point. Priority should be given to expanding the number of Māori participants within the study cohort. Being able to validate and expand upon the results we saw between Māori and non-Māori is a very interesting avenue and could prove very beneficial to the NZ healthcare system. Ideally, recruiting a larger cohort of Māori participants, with negligible traditional risk would provide interesting insights into GRS in Māori. Along a similar theme, and keeping in line with the primary aim of this study, recruiting a greater number of patients with premature MI who also have negligible traditional risk (0-1 risk factors), may also be interesting in evaluating the utility of GRS to identify young at risk patients with few additional risk features. Also, comparing all of these results to a healthy cohort of older volunteers, with no CAD, may enable us to obtain a baseline of genetic risk that could be expected in a healthy population, and therefore give a value to which the GRS of our patient population could be normalized against.

As has been mentioned previously, exploring panels with a wider or different range of SNPs will likely yield interesting results. The SNP panel we have used was shown to be effective by Mega et al, however a number of other authors have also used panels that showed utility in their respective studies $71,72,74,78,79,82-84,87,89,158$. The plethora of differing SNP panels expresses the need to analyse and consolidate these to be more robust and concordant.

\section{3 - Conclusion}

We found that GRS was not significantly elevated in the premature MI cohort versus the over 60 years MI patients. Mean GRS was significantly higher in patients with a family history of coronary disease, in Māori patients and in patients with low traditional risk. There is substantial variation in risk allele frequency between Māori and non-Māori, and between our CAD population and healthy overseas cohorts. These findings warrant further research into the genetic risk of CAD in the Māori population and into the hereditary aspects of CAD. 
Hadley Northcott 


\section{References}

1. Finegold JA, Asaria P, Francis DP. Mortality from ischaemic heart disease by country, region, and age: statistics from World Health Organisation and United Nations. Int J Cardiol 2013; 168(2): 934-45.

2. WHO. Global status report on noncommunicable diseases 2014. Switzerland: World Health Organization, 2014.

3. Mozaffarian D, Benjamin EJ, Go AS, et al. Heart disease and stroke statistics-2015 update: a report from the American Heart Association. Circulation 2015; 131(4): e29-322.

4. Health Mo. Mortality and Demographic Data 2012. Wellington: Ministry of Health; 2015.

5. Health Mo. Annual Update of Key Results 2014/15: New Zealand Health Survey. 2015.

6. Horsburgh M, Lamdin R. Maori health issues explored in an interprofessional learning context. J Interprof Care 2004; 18(3): 279-87.

7. Chan WC, Wright C, Riddell T, et al. Ethnic and socioeconomic disparities in the prevalence of cardiovascular disease in New Zealand. N Z Med J 2008; 121(1285): 11-20.

8. Harris R, Tobias M, Jeffreys M, Waldegrave K, Karlsen S, Nazroo J. Effects of selfreported racial discrimination and deprivation on Maori health and inequalities in New Zealand: cross-sectional study. Lancet 2006; 367(9527): 2005-9.

9. Westbrooke I, Baxter J, Hogan J. Are Maori under-served for cardiac interventions? N Z Med J 2001; 114(1143): 484-7.

10. Zimmerman FH, Cameron A, Fisher LD, Grace N. Myocardial infarction in young adults: Angiographic characterization, risk factors and prognosis (coronary artery surgery study registry). Journal of the American College of Cardiology 1995; 26(3): 65461.

11. Morillas P, Bertomeu V, Pabón P, et al. Characteristics and Outcome of Acute Myocardial Infarction in Young Patients. Cardiology 2007; 107(4): 217-25.

12. Hong MK, Cho SY, Hong BK, et al. Acute myocardial infarction in the young adults. Yonsei Med J 1994; 35(2): 184-9.

13. Fournier JA, Sanchez A, Quero J, Fernandez-Cortacero JAP, González-Barrero A. Myocardial infarction in men aged 40 years or less: A prospective clinical-angiographic study. Clinical Cardiology 1996; 19(8): 631-6.

14. Doughty M, Mehta R, Bruckman D, et al. Acute myocardial infarction in the young- The University of Michigan experience. American Heart Journal 2002; 143(1): $56-62$.

15. Choudhury L, Marsh JD. Myocardial infarction in young patients. The American Journal of Medicine 1999; 107(3): 254-61.

16. Abbasi SH, Kassaian SE, Sadeghian S, et al. Introducing the Tehran Heart Center's Premature Coronary Atherosclerosis Cohort: THC-PAC Study. The Journal of Tehran University Heart Center 2015; 10(1): 34-42.

17. Jamil G, Jamil M, AlKhazraji H, et al. Risk factor assessment of young patients with acute myocardial infarction. American Journal of Cardiovascular Disease 2013; 3(3): $170-4$.

18. Wong CP, Loh SY, Loh KK, Ong PJL, Foo D, Ho HH. Acute myocardial infarction: Clinical features and outcomes in young adults in Singapore. World Journal of Cardiology 2012; 4(6): 206-10.

19. Esteban MR, Montero SM, Sánchez JJA, et al. Acute Coronary Syndrome in the Young: Clinical Characteristics, Risk Factors and Prognosis. The Open Cardiovascular Medicine Journal 2014; 8: 61-7. 
20. Mohammad AM, Jehangeer HI, Shaikhow SK. Prevalence and risk factors of premature coronary artery disease in patients undergoing coronary angiography in Kurdistan, Iraq. BMC Cardiovascular Disorders 2015; 15: 155.

21. Matsis K, Holley A, Al-sinan A, Matsis P, Larsen PD, Harding SA. Differing Clinical Characteristics Between Young and Older Patients Presenting with Myocardial Infarction. Heart, Lung and Circulation 2016.

22. Health Mo. Cardiovascular Disease Risk Assessment Wellington: Ministry of Health; 2013.

23. Libby P, Theroux P. Pathophysiology of Coronary Artery Disease. Circulation 2005; 111(25): 3481-8.

24. Libby P. Inflammation in atherosclerosis. Nature 2002; 420(6917): 868-74.

25. Libby P. Mechanisms of acute coronary syndromes and their implications for therapy. $N$ Engl J Med 2013; 368(21): 2004-13.

26. Hansson GK, Libby P, Schönbeck U, Yan Z-Q. Innate and Adaptive Immunity in the Pathogenesis of Atherosclerosis. Circulation Research 2002; 91(4): 281-91.

27. Bentzon JF, Otsuka F, Virmani R, Falk E. Mechanisms of plaque formation and rupture. Circ Res 2014; 114(12): 1852-66.

28. Stocker R, Keaney JF. Role of Oxidative Modifications in Atherosclerosis. Physiological Reviews 2004; 84(4): 1381-478.

29. Li JM, Shah AM. Endothelial cell superoxide generation: regulation and relevance for cardiovascular pathophysiology. Am J Physiol Regul Integr Comp Physiol 2004; 287(5): R1014-30.

30. Stary HC, Chandler AB, Dinsmore RE, et al. A Definition of Advanced Types of Atherosclerotic Lesions and a Histological Classification of Atherosclerosis. A Report From the Committee on Vascular Lesions of the Council on Arteriosclerosis, American Heart Association 1995; 92(5): 1355-74.

31. Moore KJ, Sheedy FJ, Fisher EA. Macrophages in atherosclerosis: a dynamic balance. Nat Rev Immunol 2013; 13(10): 709-21.

32. Berliner JA, Navab M, Fogelman AM, et al. Atherosclerosis: Basic Mechanisms : Oxidation, Inflammation, and Genetics. Circulation 1995; 91(9): 2488-96.

33. Roffi M, Patrono C, Collet J-P, et al. 2015 ESC Guidelines for the management of acute coronary syndromes in patients presenting without persistent ST-segment elevation. Task Force for the Management of Acute Coronary Syndromes in Patients Presenting without Persistent ST-Segment Elevation of the European Society of Cardiology (ESC) 2016; 37(3): 267-315.

34. Thygesen K, Alpert JS, Jaffe AS, Simoons ML, Chaitman BR, White HD. Third Universal Definition of Myocardial Infarction. Circulation 2012; 126(16): 2020-35. 35. Karras C, Donlan SM, Aitchison R, Aitchison P, Wang E, Kharasch M. Acute coronary syndromes. Dis Mon 2013; 59(5): 202-9.

36. Jneid H, Anderson JL, Wright RS, et al. 2012 ACCF/AHA Focused Update of the Guideline for the Management of Patients With Unstable Angina/Non-ST-Elevation Myocardial Infarction (Updating the 2007 Guideline and Replacing the 2011 Focused Update). A Report of the American College of Cardiology Foundation/American Heart Association Task Force on Practice Guidelines 2012; 126(7): 875-910.

37. O'Gara PT, Kushner FG, Ascheim DD, et al. 2013 ACCF/AHA Guideline for the Management of ST-Elevation Myocardial InfarctionA Report of the American College of Cardiology Foundation/American Heart Association Task Force on Practice Guidelines. Journal of the American College of Cardiology 2013; 61(4): e78-e140.

38. Mahmood SS, Levy D, Vasan RS, Wang TJ. The Framingham Heart Study and the epidemiology of cardiovascular disease: a historical perspective. The Lancet 2014; 383(9921): 999-1008.

39. Alexander RW. Hypertension and the Pathogenesis of Atherosclerosis. Oxidative Stress and the Mediation of Arterial Inflammatory Response: A New Perspective 1995; 25(2): 155-61. 
40. Kuo PT. Dyslipidemia and coronary artery disease. Clin Cardiol 1994; 17(10): 519-27.

41. Nelson RH. Hyperlipidemia as a risk factor for cardiovascular disease. Prim Care 2013; 40(1): 195-211.

42. Mertens A, Holvoet P. Oxidized LDL and HDL: antagonists in atherothrombosis. FASEB J 2001; 15(12): 2073-84.

43. Stancu C, Sima A. Statins: mechanism of action and effects. Journal of Cellular and Molecular Medicine 2001; 5(4): 378-87.

44. Istvan ES, Deisenhofer J. Structural mechanism for statin inhibition of HMG-CoA reductase. Science 2001; 292(5519): 1160-4.

45. Dadu RT, Ballantyne CM. Lipid lowering with PCSK9 inhibitors. Nat Rev Cardiol 2014; 11(10): 563-75.

46. Nordestgaard BG, Chapman MJ, Ray K, et al. Lipoprotein(a) as a cardiovascular risk factor: current status. European Heart Journal 2010; 31(23): 2844-53.

47. Kamstrup PR, Tybjærg-Hansen A, Nordestgaard BG. Lipoprotein(a) and risk of myocardial infarction - genetic epidemiologic evidence of causality. Scandinavian Journal of Clinical and Laboratory Investigation 2011; 71(2): 87-93.

48. Kannel WB, McGee DL. Diabetes and cardiovascular risk factors: the Framingham study. Circulation 1979; 59(1): 8-13.

49. The Emerging Risk Factors C. Diabetes mellitus, fasting blood glucose concentration, and risk of vascular disease: a collaborative meta-analysis of 102 prospective studies. The Lancet 2010; 375(9733): 2215-22.

50. Eckel RH. Obesity and Heart Disease : A Statement for Healthcare Professionals From the Nutrition Committee, American Heart Association. Circulation 1997; 96(9): 3248-50.

51. Caballero B. The global epidemic of obesity: an overview. Epidemiol Rev 2007; 29: $1-5$.

52. Shah PK, Helfant RH. Smoking and coronary artery disease. Chest Journal 1988; 94(3): 449-52.

53. Khamis RY, Ammari T, Mikhail GW. Gender differences in coronary heart disease. Heart 2016; 102(14): 1142-9.

54. Marenberg ME, Risch N, Berkman LF, Floderus B, de Faire U. Genetic Susceptibility to Death from Coronary Heart Disease in a Study of Twins. New England Journal of Medicine 1994; 330(15): 1041-6.

55. Lloyd-Jones DM, Nam B, D'Agostino, Sr RB, et al. Parental cardiovascular disease as a risk factor for cardiovascular disease in middle-aged adults: A prospective study of parents and offspring. JAMA 2004; 291(18): 2204-11.

56. Dai X, Wiernek S, Evans JP, Runge MS. Genetics of coronary artery disease and myocardial infarction. World Journal of Cardiology 2016; 8(1): 1-23.

57. Vaidya D, Yanek LR, Moy TF, Pearson TA, Becker LC, Becker DM. Incidence of Coronary Artery Disease in Siblings of Patients With Premature Coronary Artery Disease: 10 Years of Follow-up. The American Journal of Cardiology 2007; 100(9): 14105.

58. Zdravkovic S, Wienke A, Pedersen NL, Marenberg ME, Yashin AI, De Faire U. Heritability of death from coronary heart disease: a 36-year follow-up of 20966 Swedish twins. Journal of Internal Medicine 2002; 252(3): 247-54.

59. Benedict RB. Coronary heart disease in identical female twins. The American Journal of Medicine 1958; 24(5): 814-9.

60. Slack J, Evans KA. The Increased Risk of Death from Ischaemic Heart Disease in First Degree Relatives of 121 Men and 96 Women with Ischaemic Heart Disease. Journal of Medical Genetics 1966; 3(4): 239-57.

61. Anderson AJ, Loeffler RF, Barboriak JJ, Rimm AA. Occlusive coronary artery disease and parental history of myocardial infarction. Preventive Medicine 1979; 8(3): 419-28. 
62. Nordlie MA, Wold LE, Kloner RA. Genetic contributors toward increased risk for ischemic heart disease. Journal of Molecular and Cellular Cardiology 2005; 39(4): 667-

79.

63. Austin MA, Hutter CM, Zimmern RL, Humphries SE. Familial hypercholesterolemia and coronary heart disease: a HuGE association review. Am J Epidemiol 2004; 160(5): 421-9.

64. Wang L, Fan C, Topol SE, Topol EJ, Wang Q. Mutation of MEF2A in an Inherited Disorder with Features of Coronary Artery Disease. Science 2003; 302(5650): 1578-81.

65. Bush WS, Moore JH. Chapter 11: Genome-Wide Association Studies. PLOS Computational Biology 2012; 8(12): e1002822.

66. Deloukas P, Kanoni S, Willenborg C, et al. Large-scale association analysis identifies new risk loci for coronary artery disease. Nat Genet 2013; 45(1): 25-33.

67. Hauser ER, Crossman DC, Granger CB, et al. A genomewide scan for early-onset coronary artery disease in 438 families: the GENECARD Study. Am J Hum Genet 2004; 75(3): 436-47.

68. Myocardial Infarction Genetics C, Kathiresan S, Voight BF, et al. Genome-wide association of early-onset myocardial infarction with single nucleotide polymorphisms and copy number variants. Nat Genet 2009; 41(3): 334-41.

69. Consortium CD, Nikpay M, Anuj G, et al. A comprehensive 1000 Genomes-based genome-wide association meta-analysis of coronary artery disease. Nat Genet 2015; 47(10): 1121-30.

70. Schunkert H, Konig IR, Kathiresan S, et al. Large-scale association analysis identifies 13 new susceptibility loci for coronary artery disease. Nat Genet 2011; 43(4): 333-8.

71. Abraham G, Havulinna AS, Bhalala OG, et al. Genomic prediction of coronary heart disease. European Heart Journal 2016.

72. Brautbar A, Pompeii LA, Dehghan A, et al. A genetic risk score based on direct associations with coronary heart disease improves coronary heart disease risk prediction in the Atherosclerosis Risk in Communities (ARIC), but not in the Rotterdam and Framingham Offspring, Studies. Atherosclerosis 2012; 223(2): 421-6.

73. Guella I, Duga S, Ardissino D, et al. Common variants in the haemostatic gene pathway contribute to risk of early-onset myocardial infarction in the Italian population. Thrombosis and Haemostasis 2011; 106(10): 655-64.

74. Hughes MF, Saarela 0, Stritzke J, et al. Genetic Markers Enhance Coronary Risk Prediction in Men: The MORGAM Prospective Cohorts. PLoS ONE 2012; 7(7): e40922. 75. Isaacs A, Willems SM, Bos D, et al. Risk scores of common genetic variants for lipid levels influence atherosclerosis and incident coronary heart disease. Arterioscler Thromb Vasc Biol 2013; 33(9): 2233-9.

76. Hindieh W, Pilote L, Cheema A, et al. Association Between Family History, a Genetic Risk Score, and Severity of Coronary Artery Disease in Patients With Premature Acute Coronary Syndromes. Arteriosclerosis, Thrombosis, and Vascular Biology 2016. 77. Kathiresan S, Voight BF, Purcell S, et al. Genome-wide association of early-onset myocardial infarction with single nucleotide polymorphisms and copy number variants. Nat Genet 2009; 41(3): 334-41.

78. Krarup NT, Borglykke A, Allin KH, et al. A genetic risk score of 45 coronary artery disease risk variants associates with increased risk of myocardial infarction in 6041 Danish individuals. Atherosclerosis 2015; 240(2): 305-10.

79. Labos C, Wang RHL, Pilote L, et al. Traditional risk factors and a Genetic Risk Score are associated with age of first acute coronary syndrome. Heart 2014; 100(20): 1620-4.

80. Morrison AC, Bare LA, Chambless LE, et al. Prediction of Coronary Heart Disease Risk using a Genetic Risk Score: The Atherosclerosis Risk in Communities Study. American Journal of Epidemiology 2007; 166(1): 28-35. 
81. Patel RS, Sun YV, Hartiala J, et al. Association of a Genetic Risk Score with Prevalent and Incident Myocardial Infarction in Subjects Undergoing Coronary Angiography. Circulation Cardiovascular genetics 2012; 5(4): 441-9.

82. Ripatti S, Tikkanen E, Orho-Melander M, et al. A multilocus genetic risk score for coronary heart disease: case-control and prospective cohort analyses. The Lancet 2010; 376(9750): 1393-400.

83. Tada H, Melander O, Louie JZ, et al. Risk prediction by genetic risk scores for coronary heart disease is independent of self-reported family history. European Heart Journal 2015.

84. Thanassoulis G, Peloso GM, Pencina MJ, et al. A Genetic Risk Score Is Associated With Incident Cardiovascular Disease and Coronary Artery Calcium: The Framingham Heart Study. Circulation: Cardiovascular Genetics 2012; 5(1): 113-21.

85. Tikkanen E, Havulinna AS, Palotie A, Salomaa V, Ripatti S. Genetic Risk Prediction and a 2-Stage Risk Screening Strategy for Coronary Heart Disease. Arteriosclerosis, Thrombosis, and Vascular Biology 2013; 33(9): 2261-6.

86. Vaara S, Tikkanen E, Parkkonen 0, et al. Genetic Risk Scores Predict Recurrence of Acute Coronary Syndrome. Circulation: Cardiovascular Genetics 2016.

87. Vecoli C, Adlerstein D, Shehi E, et al. Genetic score based on high-risk genetic polymorphisms and early onset of ischemic heart disease in an Italian cohort of ischemic patients. Thrombosis Research 2014; 133(5): 804-10.

88. Kathiresan S, Melander O, Anevski D, et al. Polymorphisms Associated with Cholesterol and Risk of Cardiovascular Events. New England Journal of Medicine 2008; 358(12): 1240-9.

89. Mega JL, Stitziel NO, Smith JG, et al. Genetic risk, coronary heart disease events, and the clinical benefit of statin therapy: an analysis of primary and secondary prevention trials. The Lancet 2015; 385(9984): 2264-71.

90. Berglund G, Elmstahl S, Janzon L, Larsson SA. The Malmo Diet and Cancer Study. Design and feasibility. J Intern Med 1993; 233(1): 45-51.

91. Nielsen MS, Madsen P, Christensen EI, et al. The sortilin cytoplasmic tail conveys Golgi-endosome transport and binds the VHS domain of the GGA2 sorting protein. The EMBO Journal 2001; 20(9): 2180-90.

92. Kjolby M, Nielsen MS, Petersen CM. Sortilin, Encoded by the Cardiovascular Risk Gene SORT1, and Its Suggested Functions in Cardiovascular Disease. Current Atherosclerosis Reports 2015; 17(4): 18.

93. Nykjaer A, Lee R, Teng KK, et al. Sortilin is essential for proNGF-induced neuronal cell death. Nature 2004; 427(6977): 843-8.

94. Patel KM, Strong A, Tohyama J, et al. Macrophage Sortilin Promotes LDL Uptake, Foam Cell Formation, and Atherosclerosis. Circulation research 2015; 116(5): 789-96.

95. Yung YC, Stoddard NC, Chun J. LPA receptor signaling: pharmacology, physiology, and pathophysiology. J Lipid Res 2014; 55(7): 1192-214.

96. Wilson DG, Phamluong K, Li L, et al. Global defects in collagen secretion in a Mia3/TANGO1 knockout mouse. The Journal of Cell Biology 2011; 193(5): 935-51.

97. Arndt S, Bosserhoff AK. TANGO is a tumor suppressor of malignant melanoma. International Journal of Cancer 2006; 119(12): 2812-20.

98. Sasahira T, Kirita T, Yamamoto K, et al. Transport and Golgi organisation protein 1 is a novel tumour progressive factor in oral squamous cell carcinoma. European Journal of Cancer 2014; 50(12): 2142-51.

99. Hölzel M, Rohrmoser M, Schlee M, et al. Mammalian WDR12 is a novel member of the Pes1-Bop1 complex and is required for ribosome biogenesis and cell proliferation. The Journal of Cell Biology 2005; 170(3): 367-78.

100. Rohrmoser M, Hölzel M, Grimm T, et al. Interdependence of Pes1, Bop1, and WDR12 Controls Nucleolar Localization and Assembly of the PeBoW Complex Required for Maturation of the 60S Ribosomal Subunit. Molecular and Cellular Biology 2007; 27(10): 3682-94. 
101. Moilanen A-M, Rysä J, Kaikkonen L, et al. WDR12, a Member of Nucleolar PeBoW-Complex, Is Up-Regulated in Failing Hearts and Causes Deterioration of Cardiac Function. PLoS ONE 2015; 10(4): e0124907.

102. Karnoub AE, Weinberg RA. Ras oncogenes: split personalities. Nat Rev Mol Cell Biol 2008; 9(7): 517-31.

103. Schunkert H, Erdmann J, Samani NJ. Genetics of myocardial infarction: a progress report. European Heart Journal 2010; 31(8): 918-25.

104. Tong J, Sydorskyy Y, St-Germain JR, Taylor P, Tsao MS, Moran MF. Odin (ANKS1A) Modulates EGF Receptor Recycling and Stability. PLoS ONE 2013; 8(6): e64817.

105. Allain B, Jarray R, Borriello L, et al. Neuropilin-1 regulates a new VEGF-induced gene, Phactr-1, which controls tubulogenesis and modulates lamellipodial dynamics in human endothelial cells. Cellular Signalling 2012; 24(1): 214-23.

106. Reschen ME, Lin D, Chalisey A, Soilleux EJ, O'Callaghan CA. Genetic and environmental risk factors for atherosclerosis regulate transcription of phosphatase and actin regulating gene PHACTR1. Atherosclerosis 2016; 250: 95-105.

107. Jarray R, Allain B, Borriello L, et al. Depletion of the novel protein PHACTR-1 from human endothelial cells abolishes tube formation and induces cell death receptor apoptosis. Biochimie 2011; 93(10): 1668-75.

108. Acharya A, Baek ST, Huang G, et al. The bHLH transcription factor Tcf 21 is required for lineage-specific EMT of cardiac fibroblast progenitors. Development 2012; 139(12): 2139-49.

109. Smith LT, Lin M, Brena RM, et al. Epigenetic regulation of the tumor suppressor gene TCF21 on 6q23-q24 in lung and head and neck cancer. Proceedings of the National Academy of Sciences of the United States of America 2006; 103(4): 982-7.

110. Sotiriou SN, Orlova VV, Al-Fakhri N, et al. Lipoprotein(a) in atherosclerotic plaques recruits inflammatory cells through interaction with Mac-1 integrin. The FASEB Journal 2006.

111. Gouni-Berthold I, Berthold HK. Lipoprotein(a): current perspectives. Curr Vasc Pharmacol 2011; 9(6): 682-92.

112. Tsimikas S, Witztum JL. The role of oxidized phospholipids in mediating lipoprotein(a) atherogenicity. Curr Opin Lipidol 2008; 19(4): 369-77.

113. Ichikawa T, Unoki H, Sun H, et al. Lipoprotein(a) Promotes Smooth Muscle Cell Proliferation and Dedifferentiation in Atherosclerotic Lesions of Human Apo(a) Transgenic Rabbits. The American Journal of Pathology 2002; 160(1): 227-36. 114. Ouyang T, Bai RY, Bassermann F, et al. Identification and characterization of a nuclear interacting partner of anaplastic lymphoma kinase (NIPA).J Biol Chem 2003; 278(32): 30028-36.

115. Bassermann F, von Klitzing C, Munch S, et al. NIPA defines an SCF-type mammalian E3 ligase that regulates mitotic entry. Cell 2005; 122(1): 45-57.

116. Bassermann F, von Klitzing C, Illert AL, et al. Multisite phosphorylation of nuclear interaction partner of ALK (NIPA) at G2/M involves cyclin B1/Cdk1.J Biol Chem 2007; 282(22): 15965-72.

117. Cunnington MS, Santibanez Koref M, Mayosi BM, Burn J, Keavney B.

Chromosome 9p21 SNPs Associated with Multiple Disease Phenotypes Correlate with $<$ italic>ANRIL</italic> Expression. PLoS Genet 2010; 6(4): e1000899.

118. Kotake $Y$, Nakagawa T, Kitagawa $K$, et al. Long non-coding RNA ANRIL is required for the PRC2 recruitment to and silencing of p15INK4B tumor suppressor gene. Oncogene 2011; 30(16): 1956-62.

119. Seltsam A, Hallensleben M, Kollmann A, Blasczyk R. The nature of diversity and diversification at the ABO locus. Blood 2003; 102(8): 3035-42.

120. Yamamoto F-i, Clausen H, White T, Marken J, Hakomori S-i. Molecular genetic basis of the histo-blood group ABO system. Nature 1990; 345(6272): 229-33. 
121. Sode BF, Allin KH, Dahl M, Gyntelberg F, Nordestgaard BG. Risk of venous thromboembolism and myocardial infarction associated with factor $\mathrm{V}$ Leiden and prothrombin mutations and blood type. Canadian Medical Association Journal 2013; 185(5): E229-E37.

122. De La Luz Sierra M, Yang F, Narazaki M, et al. Differential processing of stromalderived factor- $1 \alpha$ and stromal-derived factor- $1 \beta$ explains functional diversity. Blood 2004; 103(7): 2452-9.

123. Huang Y, Zhou J, Ye H, et al. Relationship between chemokine (C-X-C motif) ligand 12 gene variant (rs1746048) and coronary heart disease: Case-control study and meta-analysis. Gene 2013; 521(1): 38-44.

124. Mehta NN, Li M, William D, et al. The novel atherosclerosis locus at 10q11 regulates plasma CXCL12 levels. European Heart Journal 2011; 32(8): 963-71.

125. Askari AT, Unzek S, Popovic ZB, et al. Effect of stromal-cell-derived factor 1 on stem-cell homing and tissue regeneration in ischaemic cardiomyopathy. The Lancet 2003; 362(9385): 697-703.

126. Ghadge SK, Mühlstedt S, Özcelik C, Bader M. SDF-1 $\alpha$ as a therapeutic stem cell homing factor in myocardial infarction. Pharmacology \& Therapeutics 2011; 129(1): 97108.

127. Vasaitis TS, Bruno RD, Njar VCO. CYP17 inhibitors for prostate cancer therapy. The Journal of Steroid Biochemistry and Molecular Biology 2011; 125(1-2): 23-31. 128. Nilsson SK, Christensen S, Raarup MK, Ryan RO, Nielsen MS, Olivecrona G. Endocytosis of Apolipoprotein A-V by Members of the Low Density Lipoprotein Receptor and the Vps10p Domain Receptor Families. Journal of Biological Chemistry 2008; 283(38): 25920-7.

129. Courtois G, Morgan J, Campbell L, Fourel G, Crabtree G. Interaction of a liverspecific nuclear factor with the fibrinogen and alpha 1-antitrypsin promoters. Science 1987; 238(4827): 688-92.

130. Kleber ME, Grammer TB, Renner W, März W. Effect of the rs2259816 polymorphism in the HNF1A gene on circulating levels of c-reactive protein and coronary artery disease (the ludwigshafen risk and cardiovascular health study). $B M C$ Medical Genetics 2010; 11(1): 157.

131. Devalliere J, Charreau B. The adaptor Lnk (SH2B3): an emerging regulator in vascular cells and a link between immune and inflammatory signaling. Biochem Pharmacol 2011; 82(10): 1391-402.

132. Auburger G, Gispert S, Lahut S, et al. 12q24 locus association with type 1 diabetes: SH2B3 or ATXN2? World J Diabetes 2014; 5(3): 316-27.

133. Turner AW, Nikpay M, Silva A, et al. Functional interaction between COL4A1/COL4A2 and SMAD3 risk loci for coronary artery disease. Atherosclerosis 2015; 242(2): 543-52.

134. Olsen CL, Hsu P-P, Glienke J, Rubanyi GM, Brooks AR. Hedgehog-interacting protein is highly expressed in endothelial cells but down-regulated during angiogenesis and in several human tumors. BMC Cancer 2004; 4(1): 43.

135. Katoh Y, Katoh M. Comparative genomics on HHIP family orthologs. International Journal of Molecular Medicine 2006.

136. Liu C-j, Kong W, Ilalov K, et al. ADAMTS-7: a metalloproteinase that directly binds to and degrades cartilage oligomeric matrix protein. The FASEB Journal 2006; 20(7): 988-90.

137. Wang L, Zheng J, Bai X, et al. ADAMTS-7 Mediates Vascular Smooth Muscle Cell Migration and Neointima Formation in Balloon-Injured Rat Arteries. Circulation Research 2009; 104(5): 688-98.

138. Bauer RC, Tohyama J, Cui J, et al. Knockout of Adamts7, a novel coronary artery disease locus in humans, reduces atherosclerosis in mice. Circulation 2015; 131(13): 1202-13. 
139. Vaidyanathan G, Cismowski MJ, Wang G, Vincent TS, Brown KD, Lanier SM. The Ras-related protein AGS1//RASD1 suppresses cell growth. Oncogene 2004; 23(34): 5858-63.

140. Snow BE, Erdmann N, Cruickshank J, et al. Functional Conservation of the Telomerase Protein Est1p in Humans. Current Biology 2003; 13(8): 698-704.

141. Huntzinger E, Kashima I, Fauser M, Saulière J, Izaurralde E. SMG6 is the catalytic endonuclease that cleaves mRNAs containing nonsense codons in metazoan. RNA 2008; 14(12): 2609-17.

142. Baarends WM, Roest HP, Grootegoed JA. The ubiquitin system in gametogenesis. Molecular and Cellular Endocrinology 1999; 151(1-2): 5-16.

143. Nykjaer A, Willnow TE. The low-density lipoprotein receptor gene family: a cellular Swiss army knife? Trends in Cell Biology 2002; 12(6): 273-80.

144. Abbott GW, Sesti F, Splawski I, et al. MiRP1 Forms IKr Potassium Channels with HERG and Is Associated with Cardiac Arrhythmia. Cell 1999; 97(2): 175-87.

145. Abbott GW. KCNE2 and the K+ channel. Channels 2012; 6(1): 1-10.

146. Gabriel S, Ziaugra L, Tabbaa D. SNP Genotyping Using the Sequenom MassARRAY iPLEX Platform. Current Protocols in Human Genetics: John Wiley \& Sons, Inc.; 2001.

147. Oeth P, Beaulieu M, Park C, et al. iPLEX assay: Increased plexing efficiency and flexibility for MassArray system through single base primer extension with massmodified terminators. Sequenom application note 2005: 8876-006.

148. Kathiresan S, Srivastava D. Genetics of Human Cardiovascular Disease. Cell 2012; 148(6): 1242-57.

149. Smith JA, Ware EB, Middha P, Beacher L, Kardia SL. Current Applications of Genetic Risk Scores to Cardiovascular Outcomes and Subclinical Phenotypes. Curr Epidemiol Rep 2015; 2(3): 180-90.

150. Che R, Motsinger-Reif AA. Evaluation of genetic risk score models in the presence of interaction and linkage disequilibrium. Front Genet 2013; 4: 138.

151. Glover M, Kira A, Cowie N, Wong R, Stephen J, Marriner K. Health consequences of tobacco use for Māori-cessation essential for reducing inequalities in health. $N \mathrm{Z} \mathrm{Med}$ J 2013; 126.

152. Jin J, Li X, Gygi SP, Harper JW. Dual E1 activation systems for ubiquitin differentially regulate E2 enzyme charging. Nature 2007; 447(7148): 1135-8.

153. Aichem A, Pelzer C, Lukasiak S, et al. USE1 is a bispecific conjugating enzyme for ubiquitin and FAT10, which FAT10ylates itself in cis. Nat Commun 2010; 1: 13.

154. Nilsson SK, Heeren J, Olivecrona G, Merkel M. Apolipoprotein A-V; a potent triglyceride reducer. Atherosclerosis 2011; 219(1): 15-21.

155. Takaki S, Morita H, Tezuka Y, Takatsu K. Enhanced Hematopoiesis by Hematopoietic Progenitor Cells Lacking Intracellular Adaptor Protein, Lnk. The Journal of Experimental Medicine 2002; 195(2): 151-60.

156. Cannon CP, Battler A, Brindis RG, et al. American College of Cardiology key data elements and definitions for measuring the clinical management and outcomes of patients with acute coronary syndromesA report of the American College of Cardiology Task Force on Clinical Data Standards (Acute Coronary Syndromes Writing Committee) Endorsed by the American Association of Cardiovascular and Pulmonary Rehabilitation, American College of Emergency Physicians, American Heart Association, Cardiac Society of Australia \&amp; New Zealand, National Heart Foundation of Australia, Society for Cardiac Angiography and Interventions, and the Taiwan Society of Cardiology. Journal of the American College of Cardiology 2001; 38(7): 2114-30.

157. Armendariz AD, Krauss RM. Hepatic nuclear factor 1- $\alpha$ : inflammation, genetics, and atherosclerosis. Current Opinion in Lipidology 2009; 20(2): 106-11.

158. Ganna A, Magnusson PKE, Pedersen NL, et al. Multilocus Genetic Risk Scores for Coronary Heart Disease Prediction. Arteriosclerosis, Thrombosis, and Vascular Biology

2013; 33(9): 2267-72. 
Hadley Northcott 